\title{
Using a partial-wave method for sound-mean-flow scattering problems
}

\author{
R. Berthet \\ LPS, UMR CNRS 8550, École Normale Supérieure, 24 rue Lhomond, 75231 Paris Cedex 05, France \\ C. Coste \\ GPS, Tour 23-13, $1^{\text {er }}$ étage, 2 place Jussieu, 75251 Paris, France
}

(Received 19 September 2002; published 14 March 2003)

\begin{abstract}
We present a semianalytical method, based on a partial-wave expansion and valid in the short wavelength limit for small Mach number flows, to analyze sound-vortical-flow interactions. It is more powerful than ray-tracing methods because it gives both amplitude and phase of the sound wave, and because it is less restrictive on the smallness of the wavelength. In contrast with the Born approximation approach, this method allows the computation of the sound field in the whole interaction domain (including the near field), and preserves energy conservation. Vortical flows with finite circulation are amenable to our analysis, which gives a satisfactory description of wave front dislocation by vorticity, in good agreement with direct numerical simulations. We extend previous versions of this method to the case of smooth vorticity profiles which are observed in aeroacoustics experiments.
\end{abstract}

DOI: 10.1103/PhysRevE.67.036604

PACS number(s): 43.28. $+\mathrm{h}, 47.10 .+\mathrm{g}, 47.32 .-\mathrm{y}$

\section{INTRODUCTION}

Sound-mean-flow interactions play a prominent part in many physical phenomena, dwelling from atmospheric and oceanographic context [1] to laboratory flow instabilities [2]. It is still an open question to know how sound propagates in turbulent media, and how its characteristics are influenced by the mean flow. We will focus our attention in this paper on the short wavelength limit, when the sound wavelength $\lambda$ is smaller than the typical mean-flow scale $L$. In atmospheric and oceanographic context $[1,3,4]$, this approximation is often well verified. This limit is also useful to analyze some aspects of sound propagation in turbulent media in laboratory experiments [5-7].

In the very small wavelength limit, the acoustical interaction can be interpreted using the ray-tracing method $[4,8]$. Starting with a power law expansion of the wave amplitude in the wavelength, the zeroth order (geometric limit) leads to two coupled differential equations involving the mean-flow specifications, the wave vector $[\|\vec{k}(\tau)\|=2 \pi / \lambda(\tau)]$, and the ray path $\vec{r}(\tau)$ in terms of $\tau$, the acoustical ray parameter [9]. The solution of these sets of equations gives a qualitative description of the mean-flow effects on the geometry of the rays [10-13]. A more quantitative approach requires the acoustical field values. The first-order terms of the wavelength power law expansion lead to the conservation of the acoustical energy along a ray $[9,14]$. Thus, one can easily compute the field properties along a ray, and then estimate the spatial field repartition [15]. However, this method is no more valid in presence of caustics, when two or more rays cross: the physical requirement of a finite acoustical energy leads to a more complicated way of computation [14]. Nevertheless, the geometric limit remains a qualitative but relevant method of analysis $[16,17]$ in many problems involving sound-flow interactions.

An alternative way of solving this problem is to start with the linearized equations in the sound variables. One can then write a wave equation for the sound wave [18] with source terms expressing the coupling of sound with mean flow and solve the problem in the far-field limit in the first Born approximation $[19,20]$. This treatment, valid for small Mach number $\mathcal{M} \ll \lambda / L$, is useful in the large wavelength limit. As the wavelength decreases, $\mathcal{M} L / \lambda$ must remain small to ensure the validity of the Born approximation. Thus, the short wavelength limit cannot be easily analyzed with this method.

We will use in this paper another way of investigation: we linearize the basic equations and take the short wavelength limit. In this limit, if $\psi_{0}\left(\psi_{s}\right)$ is any quantity related to the mean flow (the sound wave), we have

$$
\psi_{s}\left\|\vec{\nabla} \psi_{0}\right\| \sim \frac{\psi_{s} \psi_{0}}{L} \ll \psi_{0}\left\|\vec{\nabla} \psi_{s}\right\| \sim k \psi_{s} \psi_{0}
$$

Then, at first order beyond the geometric limit, the sound propagation can be expressed by the modified wave equation

$$
\left(\frac{\partial}{\partial t}+\vec{v}_{0} \cdot \vec{\nabla}\right)^{2} p_{s}-c^{2} \Delta p_{s}=0
$$

where $\vec{v}_{0}$ is the mean-flow velocity, $c$ is the speed of sound and $p_{s}$ is the sound pressure perturbation. Then, an angular Fourier transform is applied to Eq. (2) and allows the complete computation of the sound field. Each Fourier mode is called a partial wave, and the complete acoustic field is the superposition (interference pattern) of all those partial waves. This method was already used for sound-mean-flow interaction problems, to study sound scattering (introducing, in analogy with scattering problems in quantum mechanics, the concept of phase shifts) [21,22], spiral waves phenomena [23], and sound absorption by a vortex [24].

The partial-wave method that we propose here is an alternative way to ray-tracing methods for sound-mean-flow scattering problem in the short wavelength limit: it allows the full computation of the acoustic fields (amplitude and phase) in the whole domain of interaction (both the far field 
and the near field). It will be applied to the scattering of a sound wave (wavelength $\lambda$ ) by a vortical stationary mean flow (typical length scale $L$ ) in the small wavelength limit $\lambda \ll L$, but in our calculations this limit is much less restrictive than in geometrical acoustics approaches.

The paper is organized as follows: in the first part, we derive a modified wave equation for the interaction of a sound wave with a mean stationary flow. In the second part, we solve this equation in the bidimensional case using the partial-wave method and we formally compute the full acoustical field. In the last part, we present examples to characterize and illustrate the method.

\section{THE WAVE EQUATION IN THE SHORT WAVELENGTH LIMIT}

Let us consider an acoustic wave (density variation $\rho_{s}$, fluid velocity $\vec{v}_{s}$, and wave celerity $c=\sqrt{p_{s} / \rho_{s}}$ ) moving in an isentropic mean flow $\left(\rho_{0}, \vec{v}_{0}\right)$. The mean flow is assumed to be incompressible, which means a small Mach number $\mathcal{M} \ll 1$, and the acoustic wave is assumed to be a small perturbation of the mean flow. Moreover, the sound frequency is assumed to be larger than the typical frequency of the mean flow, which physically means that the flow is frozen during its interaction with the sound wave.

Starting with the decomposition of the physical fields $\rho$ $=\rho_{0}+\rho_{s}, \vec{v}=\vec{v}_{0}+\vec{v}_{s}$, and $p=p_{0}+p_{s}$, we can write the mass and momentum conservation equations for the mean flow. To the leading order in the small quantities $\rho_{s} / \rho_{0}$, $v_{s} / v_{0}$, and $p_{s} / p_{0}$, the sound wave fields are governed by

$$
\begin{gathered}
\frac{D \rho_{s}}{D t}+\operatorname{div}\left(\rho_{0} \vec{v}_{s}\right)=0 \\
\rho_{0} \frac{D \vec{v}_{s}}{D t}+\rho_{0}\left(\vec{v}_{s} \cdot \overrightarrow{\operatorname{grad}}\right) \vec{v}_{0}+\rho_{s}\left(\vec{v}_{0} \cdot \overrightarrow{\operatorname{grad}}\right) \vec{v}_{0}=-c^{2} \overrightarrow{\operatorname{grad}} \rho_{s} .
\end{gathered}
$$

We have introduced the particle derivative with respect to $\vec{v}_{0}$ :

$$
\frac{D}{D t} \equiv \frac{\partial}{\partial t}+\vec{v}_{0} \cdot \overrightarrow{\mathrm{grad}}
$$

From Eqs. (3) and (4), we can write a wave equation with a source term. This source term is at least of order $\mathcal{M} / \beta^{2}$, with $\beta=k L=2 \pi L / \lambda$. In the short wavelength limit $\lambda \ll L$, which means $\beta \gg 1$, and to the first order in $\mathcal{M} \ll 1$, this source term is negligible and the wave equation becomes [23]

$$
\left[\frac{D^{2}}{D t^{2}}-c_{0}^{2} \Delta\right] \rho_{s}=0
$$

with $c_{0}$ the sound celerity in the medium at rest $\left[c \simeq c_{0}\right.$ $\left.+O\left(\mathcal{M}^{2}\right)\right]$. Then, one gets easily Eq. (2) for the sound pressure. In the following, we will focus on the density solutions of Eq. (6). Equation (6) was also derived by Obukhov for the sound velocity potential in his studies of sound scattering by turbulent flows [25].

It should be mentioned here that the wave equation must be derived first and only then the short wavelength limit be taken. Taking the short wavelength limit of Eqs. (3) and (4) and writing a wave equation can lead to a misleading physical analysis. When deriving Eq. (6), one must take the temporal derivative of Eq. (3) and the spatial derivative of Eq. (4). Then, both the wave vector $k$ and the sound frequency appear and can modify the order of magnitude of the source terms.

Equation (6), valid in the short wavelength limit for small Mach number flows, shows that the interaction between sound and flows is twofold. First, the sound wave is locally advected by the mean flow, which is a purely kinematic effect: for a uniform mean flow velocity, Eq. (6) corresponds to a Galilean transformation of the wave equation from the mean-flow reference to the laboratory reference. Second, Eq. (6) is the analogous, in a compact form due to the geometrical approximations, of the wave equation derived in the sound-mean-flow interaction studies $[18,26]$ as mentioned above. Then, the scattering by the mean-flow velocity gradients is also considered in Eq. (6).

It should be noticed that acoustical problems and surface wave problems in the shallow water approximation are similar [27] because both type of waves are nondispersive. Thus, it is not surprising that Eq. (6) is stricly analogous to the wave equation (2.7) derived by Coste et al. [28] to describe the interaction between a short wavelength surface wave and a vortical flow in the shallow water approximation.

\section{PARTIAL-WAVE METHOD IN THE SOUND SCATTERING PROBLEM}

The partial-wave (hereafter PW) method is generally used for scattering problems both in electromagnetism [29] and quantum mechanics [30] when the scatterers have a spherical or a cylindrical symmetry. In a three-dimensional problem, introducing the spherical harmonic functions allows to compute the scattering field (or the wave function) in terms of $\mathrm{PW}$, depending only on the radius $r$. In the two-dimensional case, using the polar coordinates, the PW method consists in an angular Fourier transform and also leads to PW that only depends on $r$.

In the context of sound scattering by a vortical flow, we will consider an axisymmetric bidimensional single vortex of spatial extension $L_{m}$ : its vorticity vanishes for $r \geqslant L_{m}$. Its velocity only depends on the radius $r: \vec{v}_{0}(\vec{r})=U(r) \hat{\theta}$, where $\hat{\theta}$ is the unit orthoradial vector of the polar coordinates. Assuming a small Mach number $\mathcal{M}=v_{0} / c_{0} \ll 1$ and a short wavelength with respect to the flow length $L_{m}, \beta=k L_{m}$ $=2 \pi L_{m} / \lambda \gg 1$, Eq. (6) describes the interaction of the vortical flow with the sound wave. Then, we use the PW method to compute the sound fields. In this case, the PW method simply consists in an angular Fourier transform, as pointed out before. 


\section{A. Partial-wave equation}

Since the flow is axisymmetric, Eq. (5) reads

$$
\frac{D}{D t}=\frac{\partial}{\partial t}+\frac{U(r)}{r} \frac{\partial}{\partial \theta}
$$

and Eq. (6) becomes

$$
\left[\frac{\partial^{2}}{\partial r^{2}}+\frac{1}{r} \frac{\partial}{\partial r}+\frac{1}{r^{2}} \frac{\partial^{2}}{\partial \theta^{2}}-\frac{1}{c_{0}^{2}}\left(\frac{\partial}{\partial t}+\frac{U(r)}{r} \frac{\partial}{\partial \theta}\right)^{2}\right] \rho_{s}=0 .
$$

Introducing a partial-wave development for a monochromatic sound wave of pulsation $2 \pi \nu$,

$$
\begin{aligned}
\frac{\rho_{s}(\vec{r}, t)}{\rho_{s 0}} & =\operatorname{Re}\left[\left(\sum_{n=-\infty}^{+\infty} \rho_{n}(r) e^{i n \theta}\right) e^{-i 2 \pi \nu t}\right] \\
& =\operatorname{Re}\left[\sum_{n=-\infty}^{+\infty} \rho_{n}(r) e^{i(n \theta-2 \pi \nu t)}\right],
\end{aligned}
$$

$(\operatorname{Re}[z]$ is the real part of the complex number $z)$, Eq. (8) leads, at order $\mathcal{M}$, to an ordinary differential equation for each partial wave $\rho_{n}(r)$,

$$
\rho_{n}^{\prime \prime}+\frac{\rho_{n}^{\prime}}{r}+\left[k^{2}-2 \frac{n k U(r)}{c_{0} r}-\frac{n^{2}}{r^{2}}\right] \rho_{n}=0
$$

with $k=2 \pi \nu / c_{0}$, the prime denoting a derivation with respect to $r$.

The complete solution is obtained when Eq. (10) is solved for each partial wave, and the full sound wave density variation $\rho_{s}(\vec{r}, t)$ is constructed using Eq. (9).

\section{B. Partial-wave solution}

We consider mean flows of typical vortical size $L_{m}$. We describe such a flow with a piecewise vorticity profile:

$$
\begin{gathered}
\Omega=\Omega_{\text {in }}(r), \quad r \leqslant L_{i}, \\
\Omega=\Omega_{\text {inter }}(r), \quad L_{i} \leqslant r \leqslant L_{m}, \\
\Omega=0, \quad r \geqslant L_{m} .
\end{gathered}
$$
form

The corresponding velocity can then be expressed in the

$$
\begin{gathered}
U(r)=U_{\text {in }}(r), \quad r \leqslant L_{i}, \\
U(r)=U_{\text {inter }}(r), \quad L_{i} \leqslant r \leqslant L_{m}, \\
U(r)=\frac{\Gamma}{2 \pi r}, \quad r \geqslant L_{m},
\end{gathered}
$$

$\Gamma$ being the circulation of the mean flow. We impose the continuity of the fluid velocity [and of the fluid pressure (or density)] at the boundaries between the three domains: $U_{\text {in }}\left(L_{i}\right)=U_{\text {inter }}\left(L_{i}\right)$ and $U_{\text {inter }}\left(L_{m}\right)=\Gamma /\left(2 \pi L_{m}\right)$. This structure of mean flows allows us to modelize a large number of physical situations involving axisymmetric mean flows [31-33].

\section{Outer solution}

For $r \geqslant L_{m}$, injecting the velocity field (12) in Eq. (10), we get

$$
\rho_{n}^{\prime \prime}+\frac{\rho_{n}^{\prime}}{r}+\left[k^{2}-\frac{m^{2}}{r^{2}}\right] \rho_{n}=0
$$

where $m=\sqrt{n^{2}+2 n \alpha}$ and $\alpha=k \Gamma /\left(2 \pi c_{0}\right)$. For a nonzerocirculation vortex $(\alpha \neq 0)$, the index $m$ may be real or even complex if $0>n>-2 \alpha$. In either cases, the outer solution can be expressed in terms of Bessel and Hankel functions,

$$
\rho_{n}^{\text {out }}(r)=d_{n} \frac{J_{m}(k r)}{J_{m}(\beta)}+e_{n} \frac{H_{m}^{1}(k r)}{H_{m}^{1}(\beta)},
$$

where $d_{n}$ and $e_{n}$ are two numerical constants to be determined by the boundary conditions.

In a seminal paper, Berry et al. [34] have shown the close analogy between the crossing of a potential vector of finite circulation by a charged particle in quantum mechanics and the interaction between a surface wave and a vortical flow in an ordinary fluid. The vortex has the same effect on the surface waves as the vector potential on the wave function: the finite circulation implies a phase shift, characterized by the parameter $\alpha$, in either the wave function or the surface wave, and the wave fronts display a dislocation (the so-called Aharonov-Bohm effect in quantum mechanics [35]). This dislocated wave is a non perturbative interaction term (the dislocation amplitude is the same as that of the wave), and persists arbitrarily far from the interaction region, which is not the case for the scattered wave (with amplitude decreasing as $1 / \sqrt{r}$ in two dimensions).

The wave front dislocation is a phase shift that cannot be directly observed in quantum mechanics [36], whereas in the acoustical or surface waves cases, both the phase and amplitude of the waves can be measured: the Berry's analogy was experimentally confirmed by Vivanco et al. [37] in the surface wave case and in the acoustical case by Roux et al. [31] using the time reversal mirror method and by Labbé and Pinton [32] with direct ultrasonic measurements.

The dislocation parameter $\alpha$ is zero when the vortex has no circulation. It leads to Bessel functions of integer order in solution (14): there is no Aharonov-Bohm effect in this case. Thus, in classical wave scattering experiments, a dislocated wave front can be interpreted as the signature of a vortical flow with a global circulation $[37,38]$.

\section{Inner solution}

In the vortex region, we must solve

$$
\rho_{n}^{\prime \prime}+\frac{\rho_{n}^{\prime}}{r}+\left[k^{2}-2 \frac{n k U_{\text {inter }}(r)}{c_{0} r}-\frac{n^{2}}{r^{2}}\right] \rho_{n}=0
$$


for $L_{i} \leqslant r \leqslant L_{m}$ and

$$
\rho_{n}^{\prime \prime}+\frac{\rho_{n}^{\prime}}{r}+\left[k^{2}-2 \frac{n k U_{i n}(r)}{c_{0} r}-\frac{n^{2}}{r^{2}}\right] \rho_{n}=0
$$

for $r \leqslant L_{i}$.

In the region $r \leqslant L_{i}, U_{i n} / r$ is an angular velocity: it must remain finite when $r$ goes to zero. Thus, in this limit, $U_{i n} / r \ll n^{2} / r^{2}$ and Eq. (16) always becomes a usual Bessel equation: its solution is a linear combination of Bessel and Neumann functions $J$ and $N$. When $r$ goes to zero, the Neumann function diverges, and so does one of the solutions of Eq. (16). This latter must consequently be excluded, in order to get a finite sound field everywhere. The inner solution can thus be expressed in a formal way in both regions, using a set of only three numerical constants $a_{n}, b_{n}$, and $c_{n}$ :

$$
\rho_{n}^{i n}(r)=a_{n} \frac{F_{n}^{i n}(r)}{F_{n}^{i n}\left(L_{i}\right)},
$$

where $F_{n}^{i n}(r)$ is that solution of Eq. (16) which is bounded at the origin $r=0$, and

$$
\rho_{n}^{\text {inter }}(r)=b_{n} \frac{F_{n}^{\text {inter }}(r)}{F_{n}^{\text {inter }}\left(L_{m}\right)}+c_{n} \frac{G_{n}^{\text {inter }}(r)}{G_{n}^{\text {inter }}\left(L_{m}\right)} .
$$

The explicit form of the three functions $F_{n}^{i n}, F_{n}^{\text {inter }}$, and $G_{n}^{\text {inter }}$ obviously depends on the details of the velocity profile.

\section{Matching of the three solutions}

To achieve the determination of the sound wave, we have to compute the five integration constants $a_{n}, b_{n}, c_{n}, d_{n}$, and $e_{n}$. They are fixed by requiring the continuity of both the density variation and the fluid velocity for the sound wave at the matching locations $r=L_{i}$ and $r=L_{m}$. This implies the continuity of $\rho_{n}$ for each PW, and from Eq. (4) the velocity continuity leads to that of $\rho_{n}^{\prime}$. This provides a set of four equations.

The fifth equation comes from a topological condition on the sound field at infinity: as pointed out before, far from the mean flow, $k r \rightarrow \infty$, the sound wave should be the sum [39] of a scattered wave, decreasing like $1 / \sqrt{k r}$, and a dislocated wave of constant amplitude [28,34]. This condition reads [34]

$$
d_{n}=(-i)^{m} J_{m}(\beta)
$$

and the continuity conditions lead to the nonhomogeneous system

$$
\left(\begin{array}{cccc}
1 & -\frac{F_{n}^{\text {inter }}\left(L_{i}\right)}{F_{n}^{\text {inter }}\left(L_{m}\right)} & -\frac{G_{n}^{\text {inter }}\left(L_{i}\right)}{G_{n}^{\text {inter }}\left(L_{m}\right)} & 0 \\
\frac{\left(F_{n}^{\text {in }}\right)^{\prime}\left(L_{i}\right)}{F_{n}^{\text {in }}\left(L_{i}\right)} & -\frac{\left(F_{n}^{\text {inter }}\right)^{\prime}\left(L_{i}\right)}{F_{n}^{\text {inter }}\left(L_{m}\right)} & -\frac{\left(G_{n}^{\text {inter }}\right)^{\prime}\left(L_{i}\right)}{G_{n}^{\text {inter }}\left(L_{m}\right)} & 0 \\
0 & 1 & 1 & -1 \\
0 & \frac{\left(F_{n}^{\text {inter }}\right)^{\prime}\left(L_{m}\right)}{F_{n}^{\text {inter }}\left(L_{m}\right)} & \frac{\left(G_{n}^{\text {inter }}\right)^{\prime}\left(L_{m}\right)}{G_{n}^{\text {inter }}\left(L_{m}\right)} & -\frac{H_{m}^{\prime 1}(\beta)}{H_{m}^{1}(\beta)}
\end{array}\right)=\left(\begin{array}{c}
a_{n} \\
b_{n} \\
c_{n} \\
e_{n}
\end{array}\right)=\left(\begin{array}{c}
0 \\
0 \\
(-i)^{m} J_{m}(\beta) \\
(-i)^{m} J_{m}^{\prime}(\beta)
\end{array}\right)
$$

Then, the complete solution $\rho_{s}$ is

$$
\begin{aligned}
\frac{\rho_{s}(r, \theta, t)}{\rho_{s 0}}= & \operatorname{Re}\left[\sum_{n=-\infty}^{\infty} a_{n} \frac{F_{n}^{i n}(r)}{F_{n}^{i n}\left(L_{i}\right)} e^{i(n \theta-2 \pi \nu t)}\right], \quad r \leqslant L_{i}, \\
\frac{\rho_{s}(r, \theta, t)}{\rho_{s 0}}= & \operatorname{Re}\left[\sum _ { n = - \infty } ^ { \infty } \left(b_{n} \frac{F_{n}^{\text {inter }}(r)}{F_{n}^{\text {inter }}\left(L_{m}\right)}\right.\right. \\
& \left.\left.+c_{n} \frac{G_{n}^{\text {inter }}(r)}{G_{n}^{\text {inter }}\left(L_{m}\right)}\right) e^{i(n \theta-2 \pi \nu t)}\right], \quad L_{i} \leqslant r \leqslant L_{m},
\end{aligned}
$$

$$
\begin{aligned}
\frac{\rho_{s}(r, \theta, t)}{\rho_{s 0}}= & \operatorname{Re}\left[\sum _ { n = - \infty } ^ { \infty } \left((-i)^{m} J_{m}(k r)\right.\right. \\
& \left.\left.+e_{n} \frac{H_{m}^{1}(k r)}{H_{m}^{1}(\beta)}\right) e^{i(n \theta-2 \pi \nu t)}\right], \quad r \geqslant L_{m},
\end{aligned}
$$

where the constants $a_{n}, b_{n}, c_{n}$, and $e_{n}$ are given by the solution of Eq. (20). All our PW calculations are done using MATHEMATICA. The symbolic computation capabilities of this software are useful to calculate the constants.

In the limit $\alpha \rightarrow 0, m=|n|$ and the outside sound wave is the exact sum of the incident plane wave and a wave scattered by a cylindrical distribution [40]. It should be pointed 
out that we take here into account the inner structure of this distribution [see Eq. (20)].

\section{Scattering amplitude}

Assuming a linear incident plane wave of wave number $k$, wavelength $\lambda=2 \pi / k$, celerity $c$, frequency $\nu=\lambda / c$, and density amplitude $\rho_{0 i}$,

$$
\rho_{i}=\rho_{0 i} \operatorname{Re}\left[e^{i(\vec{k} \cdot \vec{r}-2 \pi \nu t)}\right],
$$

two quantities of major importance for sound scattering studies can be defined [40]: the scattered sound wave $\rho_{\text {scat }}$ and the scattered amplitude $f(\theta)$. The former is deduced from $\rho_{s}$ and $\rho_{i}$ :

$$
\rho_{s} \equiv \rho_{i}+\rho_{\text {scat }}
$$

and the latter, only defined for $r \gg 2 \pi L^{2} / \lambda$ (far-field approximation), is deduced from $\rho_{\text {scat }}$ and $\rho_{i}$ by

$$
\rho_{\text {scat }}=\operatorname{Re}\left[\rho_{0 i} f(\theta) e^{-i(2 \pi \nu t)} \frac{e^{i k r}}{\sqrt{r}}\right]
$$

in two dimensions. The $1 / \sqrt{r}$ factor in Eq. (26) comes from the energy conservation in a two-dimensional problem. $f(\theta)$ characterizes the angular structure of the sound wave resulting from the scattering process. It is directly related to the scattering cross section:

$$
\sigma_{\text {scat }}=\int_{0}^{2 \pi}|f(\theta)|^{2} d \theta
$$

an important parameter to analyze the scattering efficiency.

The wave front dislocation is a nonperturbative effect due to the finite circulation of the vortical flow at infinity. Hence for a compact mean flow of typical size $L$ (no velocity circulation for $r \geqslant L$ ), there is no dislocated wave, and the incident plane wave is the exact solution of the scattering problem far from the vortex. In the context of our calculations, $\alpha=0, m(n, \alpha)=|n|$ where $n$ is an integer, and Eq. (19) leads to the classical decomposition of plane waves in series of Bessel functions (see Ref. [41], formula (8.511) and notice that this choice implies to locate the forward direction in $\theta$ $=\pi)$ :

$$
\begin{aligned}
\frac{\rho_{i}}{\rho_{0 i}} & =\operatorname{Re}\left[e^{i(\vec{k} \cdot \vec{r}-2 \pi \nu t)}\right] \\
& =\operatorname{Re}\left[e^{-i(2 \pi \nu t)} \sum_{n=-\infty}^{\infty}(-i)^{n} e^{i n \theta} J_{n}(k r)\right] .
\end{aligned}
$$

Comparing this last expression with the expression of the outer solution (23), we conclude that the scattered wave $\rho_{\text {scat }}$ corresponds to the second term of the partial-wave solution:

$$
\frac{\rho_{\text {scat }}(r, \theta, t)}{\rho_{0 i}}=\operatorname{Re}\left[\sum_{n=-\infty}^{\infty} e_{n} \frac{H_{m}^{1}(k r)}{H_{m}^{1}(\beta)} e^{i(n \theta-2 \pi \nu t)}\right] .
$$

Far from the vortex core, $k r \gg 1$, we can expand in $k r$ the Hankel functions $H_{m}^{1}$ :

$$
H_{m}^{1}(k r) \simeq(-i)^{m} \sqrt{\frac{2}{\pi k r}} e^{i k r} e^{-i \pi / 4}
$$

We recover the $e^{i k r} / \sqrt{r}$ radial evolution, and can express the scattering amplitude $f(\theta)$ as

$$
f(\theta)=\sqrt{\frac{2}{\pi k}} \sum_{n=-\infty}^{\infty} e_{n} \frac{e^{i(n \theta-\pi / 4-m \pi / 2)}}{H_{m}^{1}(\beta)} .
$$

The scattering cross section can then be formally computed in terms of the partial wave coefficients $e_{n}$ :

$$
\sigma_{\text {scat }}=\frac{4}{k} \sum_{n=-\infty}^{\infty}\left|\frac{e_{n}}{H_{m}^{1}(\beta)}\right|^{2} .
$$

\section{Conservation of the acoustical energy}

Another way to compute $\sigma_{\text {scat }}$ is by using the twodimensionnal optical theorem $[29,42]$

$$
\sigma_{s c a t}+\sigma_{a b s}=\sigma_{t}=\sqrt{\frac{8 \pi}{k}} \operatorname{Im}\left[f(\theta=\pi) e^{-i \pi / 4}\right]
$$

( $\theta=\pi$ locates the forward direction, see Eq. (28) and $\operatorname{Im}(z)$ is the imaginary part of the complex number $z$ ).

It simply expresses the energy conservation during the scattering process. As we do not consider any source of absorption, $\sigma_{a b s}=0$ and we expect

$$
\sigma_{s c a t}=\sigma_{t}
$$

From Eqs. (31) and (32), it is a very hard task to demonstrate this equality because of the cumbersome expression of the coefficients $e_{n}$. Nevertheless, the quantum problem formally analogous to our acoustical problem is the interaction between a particle and an axisymmetrical potential $V(r)$ : starting with the Schrödinger equation [30]

$$
\Delta \Psi+\frac{2 m}{\hbar^{2}}[E-V(r)] \Psi=0,
$$

one can introduce a partial-wave development of the wave function $\Psi$,

$$
\Psi(\vec{r})=\sum_{n=-\infty}^{+\infty} \Psi_{l}(r) e^{i l \theta} .
$$

Then, each partial wave $\Psi_{l}$ satisfies

$$
\Psi_{l}^{\prime \prime}+\frac{\Psi_{l}^{\prime}}{r}+\left[k^{2}-2 \frac{m V(r)}{\hbar^{2}}-\frac{l^{2}}{r^{2}}\right] \Psi_{l}=0 .
$$

Equation (37) is similar to Eq. (10). Moreover, if we consider a compact mean flow $U(r)$ in acoustics, its quantum analog is an interaction potential $V(r)$ which decays faster than $1 / r$ [43]. In the large $r$ region (corresponding to the far 
field in acoustics and to the large distance scattering in quantum mechanics), both equations have the same asymptotic limits

$$
X^{\prime \prime}+k^{2} X=0 \quad\left[X(r) \equiv \sqrt{r} \rho_{n}(r) \operatorname{or} \sqrt{r} \Psi_{l}(r)\right] .
$$

We use this quantum mechanics analogy to solve our scattering problem: far from the scattering region [43],

$$
\rho_{n} \propto \sqrt{\frac{2}{\pi k r}} \cos \left(k r-n \pi / 2+\delta_{n}\right),
$$

where the phase shift $\delta_{n}$ depends on the velocity field $U(r)$ [for $U \equiv 0, \delta_{n} \equiv 0$ and one gets the asymptotic expansion of the partial waves describing a monochromatic plane wave, Eq. (42)]. Then the complete sound wave $\rho_{s}$ reads

$$
\begin{aligned}
\frac{\rho_{s}(r, \theta, t)}{\rho_{0 i}}= & \sqrt{\frac{2}{\pi k r}} \operatorname{Re}\left[\sum_{n=-\infty}^{\infty} B_{n} \cos (k r-n \pi / 2\right. \\
& \left.\left.+\delta_{n}\right) e^{i(n \theta-2 \pi \nu t)}\right]
\end{aligned}
$$

where $B_{n}$ are numerical constants. On the other hand, from Eqs. (24) $-(26), \rho_{s}$ can be expressed as

$$
\frac{\rho_{s}(r, \theta, t)}{\rho_{0 i}}=\operatorname{Re}\left[e^{i(\vec{k} \cdot \vec{r}-2 \pi \nu t)}+\frac{f(\theta)}{\sqrt{r}} e^{i(k r-2 \pi \nu t)}\right]
$$

and the incident plane wave can be expanded into [see Eq. (28) and Ref. [41], formula (8.451)]

$$
\begin{aligned}
\frac{\rho_{i}}{\rho_{0 i}}= & \operatorname{Re}\left[e^{i(\vec{k} \cdot \vec{r}-2 \pi \nu t)}\right] \\
= & \sqrt{\frac{2}{\pi k r}} \operatorname{Re}\left[e^{-i(2 \pi \nu t)} \sum_{n=-\infty}^{\infty}(-i)^{n}\right. \\
& \left.\times e^{i n \theta} \cos (k r-n \pi / 2-\pi / 4)\right] .
\end{aligned}
$$

The constants $B_{n}$ are chosen in such a way that the scattered wave $\rho_{s}-\rho_{i}$ represents an outgoing wave, depending only on $e^{+i k r}$. Using Eqs. (40)-(42) and equating to 0 the coefficient of $e^{-i k r}$ lead to

$$
B_{n}=e^{i\left(\delta_{n}-n \pi / 2+\pi / 4\right)}
$$

and the scattering amplitude can be recast into

$$
f(\theta)=\sqrt{\frac{1}{2 \pi k}} \sum_{n=-\infty}^{\infty} e^{i n(\theta-\pi)} e^{-i \pi / 4}\left[e^{i\left(2 \delta_{n}+\pi / 2\right)}-1\right] .
$$

Similar expressions were derived for the scattering of sound by nonzero-circulation mean flows by Fetter in the large wavelength limit $\lambda \gg L$ [21], and by Reinschke [22]. However, these authors did not discuss the validity of the optical theorem because they considered problems in which the mean-flow velocity field decays too slowly.

From Eq. (31), we get a complete analogy between our partial-wave method and the phase-shift method:

$$
e_{n}=\frac{H_{m}^{1}(\beta) e^{i(m \pi / 2)}}{2} e^{-i n \pi}\left[e^{i\left(2 \delta_{n}+\pi / 2\right)}-1\right] .
$$

From Eq. (44), we can compute the scattering cross section $\sigma_{\text {scat }}$

$$
\sigma_{\text {scat }}=\frac{4}{k} \sum_{n=-\infty}^{\infty} \sin ^{2}\left(\delta_{n}+\pi / 4\right),
$$

and the forward scattering amplitude

$$
f(\theta=\pi)=e^{-i \pi / 4} \sqrt{\frac{1}{2 \pi k}} \sum_{n=-\infty}^{\infty}\left[e^{i\left(2 \delta_{n}+\pi / 2\right)}-1\right] .
$$

Thus, we prove the conservation of the acoustical energy (33) and find $\sigma_{\text {scat }}=\sigma_{t}$.

Therefore, the partial-wave analysis of Eq. (6), valid at the first Mach order in the short wavelength, includes the conservation of the acoustical energy during the scattering process. If the interaction is analyzed in the first Born approximation, it is well known that the optical theorem cannot be satisfied: $f \propto \mathcal{M}$ whereas $\sigma \propto \mathcal{M}^{2}$. The second Born approximation $[30,44]$ or an asymptotical treatment $[45,46]$ must be considered to ensure energy conservation.

Energy conservation is satisfied here because we solve exactly Eq. (6), taking into account the full interaction phenomena, including both multiscattering events and geometrical coupling. The consequence is that our calculation fulfills the optical theorem for a compact mean flow (quickly decreasing at infinity), and that it describes properly the nonpertubative effect of wave front dislocation for a noncompact mean flow. This is clearly not the case in the first Born approximation or the standard ray-tracing methods.

\section{APPLICATION TO AEROACOUSTIC PROBLEMS}

The PW method has been employed to solve the diffraction of surface waves by a single vortex [37]. In this context, the experimental vorticity distribution is very accurately described by a vortex core in solid rotation, and an outer part of the flow with a $1 / r$ decreasing orthoradial velocity (see Appendix A). A very good agreement was found between experimental results and the PW method predictions [37,47]. Formally, the vorticity is a step function of the distance to the vortex center. In aeroacoustics, however, the solid rotation is a very poor approximation of the effective velocity field in the inner part of the vortex [32]. We thus show, in these examples, how to generalize the previous calculations [28] to smooth (polynomial) vorticity distributions.

Another difficulty with the PW method is that the basic equation (6) is only approximate, valid when $\beta \gg 1$ where $\beta$ is the product of the acoustic wave number by a characteristic length scale of the flow, typically the size of the vorticity distribution. But the analysis does not give any numerical 
estimate of the minimum admissible value of $\beta$. In order to get more information, we study the acoustic scattering by a vorticity distribution of zero circulation outside a radius $L_{m}$. In this case, the scattered wave may be calculated in the Born approximation (see Appendix B), which is valid if $\mathcal{M} \beta \ll 1$ where $\mathcal{M}$ is the flow Mach number $[48,49]$. It is thus possible to consider low Mach number flows for which the Born approximation is legitimate, and to compare the Born scattering amplitude with PW calculations. Then, we get numerical estimates of the value of $\beta$ that gives good numerical agreement between the two approaches.

\section{A. Range of validity of the partial-wave method}

To analyze the range of validity of our method, we want to make comparisons with usual calculations in the first Born approximation. To this end, we use two zero-circulation flows, because Born approximation breaks down in the case of flows with circulation [19,50,51]. Setting $b_{i}$ the vortex size and $a_{i}=x_{i} b_{i}\left(x_{i}<1\right)$, the first one is

$$
\begin{gathered}
U_{1}(r)=\frac{\omega_{1}}{2} r, \quad 0 \leqslant r \leqslant a_{1}, \\
U_{1}(r)=\frac{\omega_{1} x_{1}^{2}}{2\left(x_{1}^{2}-1\right)}\left(r-\frac{b_{1}^{2}}{r}\right), \quad a_{1} \leqslant r \leqslant b_{1}, \\
U_{1}(r)=0, \quad b_{1}<r . \\
\Omega_{1}(r)=\omega_{1}, \quad 0 \leqslant r \leqslant a_{1}, \\
\Omega_{1}(r)=\frac{\omega_{1} x_{1}^{2}}{x_{1}^{2}-1}, \quad a_{1} \leqslant r \leqslant b_{1}, \\
\Omega_{1}(r)=0, \quad b_{1}<r .
\end{gathered}
$$

The velocity field is continuous at $r=a_{1}$, but the vorticity is not. This is corrected in the second case,

$$
\begin{gathered}
U_{2}(r)=\frac{\omega_{2}}{2} r\left(1-\frac{r^{2}}{x_{2}^{2} b_{2}^{2}\left(2-x_{2}^{2}\right)}\right), \quad 0 \leqslant r \leqslant a_{2}, \\
U_{2}(r)=\frac{\omega_{2} x_{2}^{2}}{2\left(2-x_{2}^{2}\right)} r\left(\frac{b_{2}^{2}}{r^{2}}-1\right), \quad a_{2} \leqslant r \leqslant b_{2}, \\
U_{2}(r)=0, \quad b_{2}<r . \\
\Omega_{2}(r)=\omega_{2}\left(\begin{array}{l}
\left.1-\frac{2 r^{2}}{x_{2}^{2} b_{2}^{2}\left(2-x_{2}^{2}\right)}\right), \quad 0 \leqslant r \leqslant a_{2}, \\
\Omega_{2}(r)=-\omega_{2} \frac{x_{2}^{2}}{2-x_{2}^{2}}, \quad a_{2} \leqslant r \leqslant b_{2}, \\
\Omega_{2}(r)=0, \quad b_{2}<r .
\end{array}\right.
\end{gathered}
$$

This second velocity profile is thus smooth, which is in principle a more favorable situation since the velocity gradients are lower.

The analytical expressions of the velocity fields are chosen to make the PW calculations easier. In order to facilitate the comparison, we impose the same flow size $b_{1}=L_{m}=b_{2}$ and we determine the value of the parameter $\omega_{2}\left(\omega_{1}\right.$ fixes the velocity scale) by the following physical requirement: we require for the two flows the same maximal velocity, which means the same Mach number, and that this maximum velocity occurs at the same radius $r=L^{*}$.

Straightforward algebra leads to

$$
\begin{gathered}
a_{1}=L^{*}, \\
a_{2}=b_{2}\left(1-\sqrt{1-3 \frac{L^{* 2}}{b_{2}^{2}}}\right)^{1 / 2} \\
\omega_{2}=\frac{3 \omega_{1}}{2} .
\end{gathered}
$$

In the following, we compare the scattering amplitudes $f$ defined by Eq. (26), computed using either the first Born approximation applied directly on Eq. (6) or the PW solution, Eq. (31).

\section{Partial-wave computations}

With the first vortical flow $U_{1}$, Eqs. (15) and (16) are Bessel equations, with solutions

$$
\begin{gathered}
F_{n}^{\text {in }}(r)=J_{|n|}\left(\sqrt{k k_{n}^{0}} r\right), \quad F_{n}^{\text {inter }}(r)=J_{p}\left(\sqrt{k k_{n}^{1}} r\right), \\
G_{n}^{\text {inter }}(r)=N_{p}\left(\sqrt{k k_{n}^{1}} r\right),
\end{gathered}
$$

with

$$
\begin{gathered}
k_{n}^{0}=k-n \omega_{1} / c_{0}, \quad k_{n}^{1}=k-\frac{n}{c_{0}} \frac{\omega_{1} x_{1}^{2}}{x_{1}^{2}-1}, \\
p^{2}=n^{2}-\frac{n k}{c_{0}} \frac{\omega_{1} x_{1}^{2} L_{m}^{2}}{x_{1}^{2}-1} .
\end{gathered}
$$

In the region $0 \leqslant r<a_{1}$, we have kept the Bessel function of positive index only, since it is the only solution of Bessel equation which is finite for $r=0$.

If $c_{0} k / \omega_{1}$ is an integer $n_{0}, k_{n}^{0}$ vanishes for $n=n_{0}$. In this special case, the inner solution can be found by assuming $F_{n_{0}}^{i n}(r) \propto r^{p}$. This leads to $p= \pm n_{0}$ and we only keep the solution $p=n_{0}$ to ensure a bounded solution at the origin:

$$
F_{n_{0}}^{i n}(r)=r^{n_{0}}
$$

If $k_{n}^{1}$ vanishes for some $n=n_{1}$, the intermediate solution can also be found $\propto r^{p}$. This leads to $p= \pm n_{1}$ and the intermediate solution becomes, for this particular value of $n$,

$$
F_{n_{1}}^{\text {inter }}(r)=r^{n_{1}}, \quad G_{n_{1}}^{\text {inter }}(r)=r^{-n_{1}} .
$$


For the second vortical flow $U_{2}$, Eq. (15) is still a Bessel equation with solutions

$$
F_{n}^{\text {inter }}(r)=J_{p}\left(\sqrt{k k_{n}^{2}} r\right), \quad G_{n}^{\text {inter }}(r)=N_{p}\left(\sqrt{k k_{n}^{2}} r\right)
$$

with

$$
k_{n}^{2}=k+\frac{n}{c_{0}} \frac{\omega_{2} x_{2}^{2}}{2-x_{2}^{2}}, \quad p^{2}=n^{2}+\frac{2 n k L_{m}^{2}}{c_{0}} \frac{\omega_{2} x_{2}^{2}}{2-x_{2}^{2}} .
$$

Again, if $k_{n}^{2}$ vanishes for some $n=n_{2}$, the intermediate solution becomes

$$
F_{n_{2}}^{\text {inter }}(r)=r^{n_{2}}, \quad G_{n_{2}}^{\text {inter }}(r)=r^{-n_{2}} .
$$

We have chosen the vortical flow $U_{2}$ in order that Eq. (16) becomes a solvable equation. Bose [52] has given a change of variable that links it to the Wittacker equation [see Ref. [41], formula (9.220.1)], and its solutions are expressed in terms of confluent hypergeometric functions ${ }_{1} F_{1}$ (also called degenerate hypergeometric functions; see Ref. [41], Sec. 9.21):

$$
\begin{aligned}
F_{n}^{i n}(r)= & \frac{\left[c\left(r / a_{2}\right)^{2}\right]^{\mu}}{\sqrt{2}} \exp \left[-c\left(r / a_{2}\right)^{2} / 2\right]_{1} F_{1}(1 / 2+\mu-\lambda, 1 \\
& \left.+2 \mu, c\left(r / a_{2}\right)^{2}\right)
\end{aligned}
$$

with

$$
c=a_{2}^{2} \sqrt{\frac{n k \omega_{2}}{c_{o} a_{2}^{2}\left(2-x_{2}^{2}\right)}}, \quad \mu=\frac{|n|}{2}, \quad \lambda=\frac{a_{2}^{2}}{4 c}\left(k^{2}-\frac{n k \omega_{2}}{c_{0}}\right) .
$$

As before, we have kept the only solution that is finite at $r$ $=0$.

If $c_{0} k / \omega_{2}$ is an integer $n_{3}, \lambda$ vanishes for $n=n_{3}$ and Eq. (16) becomes a Bessel equation. Nevertheless, the inner solution can still be expressed in terms of confluent hypergeometric functions, using the relation [52]

$$
J_{\mu}(z)=\frac{1}{\Gamma(\mu+1)}\left(\frac{z}{2}\right)^{\mu} \exp (-i z)_{1} F_{1}(1 / 2+\mu, 1+2 \mu, 2 i z) .
$$

For the particular value $n=0, c \equiv 0$ and $\mu \equiv 0$, and the solution (62) are no longer valid. In this case, Eq. (16) becomes a Bessel equation and the inner solution, finite at $r=0$, is

$$
F_{0}^{i n}(r)=J_{0}(k r) .
$$

Then the scattering amplitude (31), which requires the computation of the constants $e_{n}$, can be expressed in both cases by injecting the particular functions (55) or (59) and (62) in the general system (20).

\section{Born approximation}

From Appendix B, since we consider only axisymmetric vortical flows, we need to compute

$$
\widetilde{\Omega}(\vec{q})=2 \pi \int_{0}^{\infty} \Omega(r) J_{0}(q r) r d r .
$$

Injecting the vorticity fields (49) and (51), we calculate the two Fourier transforms

$$
\begin{aligned}
\widetilde{\Omega}_{1}(\vec{q})= & \omega_{1} \frac{2 \pi}{q}\left(a_{1} J_{1}\left(q a_{1}\right)+\frac{x_{1}^{2}}{\left(x_{1}^{2}-1\right)}\right. \\
& \left.\times\left[b_{1} J_{1}\left(q b_{1}\right)-a_{1} J_{1}\left(q a_{1}\right)\right]\right)
\end{aligned}
$$

and

$$
\begin{aligned}
\widetilde{\Omega}_{2}(\vec{q})= & \omega_{2} \frac{2 \pi}{q}\left(\left[a_{2} J_{1}\left(q a_{2}\right)-\frac{+2}{\left(2-x_{2}^{2}\right) q}\left[2 J_{2}\left(q a_{2}\right)\right.\right.\right. \\
& \left.\left.-q a_{2} J_{3}\left(q a_{2}\right)\right]\right]-\frac{x_{2}^{2}}{2-x_{2}^{2}}\left[b_{2} J_{1}\left(q b_{2}\right)\right. \\
& \left.\left.-a_{2} J_{1}\left(q a_{2}\right)\right]\right)
\end{aligned}
$$

and then compute the scattering amplitude with the help of relation $(\mathrm{B} 1)$.

\section{Estimation of the range of validity}

For a mean flow of size $L_{m}, \beta=2 \pi L_{m} / \lambda$ is the control parameter of the PW method. By construction, both flows correspond to the same value of $\beta$. For each value of $\beta$, and each flow, we compute the scattering amplitude from the PW method $f^{P W}(\theta)$ and from the first Born approximation $f^{B}(\theta)$. Then, we compute the scattering cross sections $\sigma_{\text {scat }}^{P W}$ from Eq. (32) and $\sigma_{\text {scat }}^{B}$ from Eq. (27) and analyze the error between the two methods with the error parameter

$$
\zeta=\frac{2\left(\sigma_{\text {scat }}^{P W}-\sigma_{\text {scat }}^{B}\right)}{\sigma_{\text {scat }}^{P W}+\sigma_{\text {scat }}^{B}} .
$$

For the Born approximation to be valid, the flow must satisfy $\mathcal{M} \beta=2 \pi \mathcal{M} L_{m} / \lambda \ll 1$. Thus, we choose $\mathcal{M}=10^{-4}$ to ensure that this inequality is satisfied for large values of $\beta$.

Figure 1 shows the evolution of the error $\zeta$ for the two flows. Obviously, $\zeta$ decreases as $\beta$ increases. Moreover, the error values depend very slightly on the flow shape and on its smoothness. From a more quantitative point of view, the PW method remains valid for $\beta \geqslant 10(\zeta \leqslant 10 \%)$. For very accurate computations, one must choose $\beta \geqslant 20$ to ensure $\zeta$ $\leqslant 1 \%$.

\section{B. Examples}

It is obvious that analytical solutions of Eqs. (15) and (16) are obtainable with few particular mean-flow velocity fields only. In the simplest case, analytical solutions can be expressed in terms of Bessel functions if $U \propto r$ or $U \propto 1 / r$ (see 


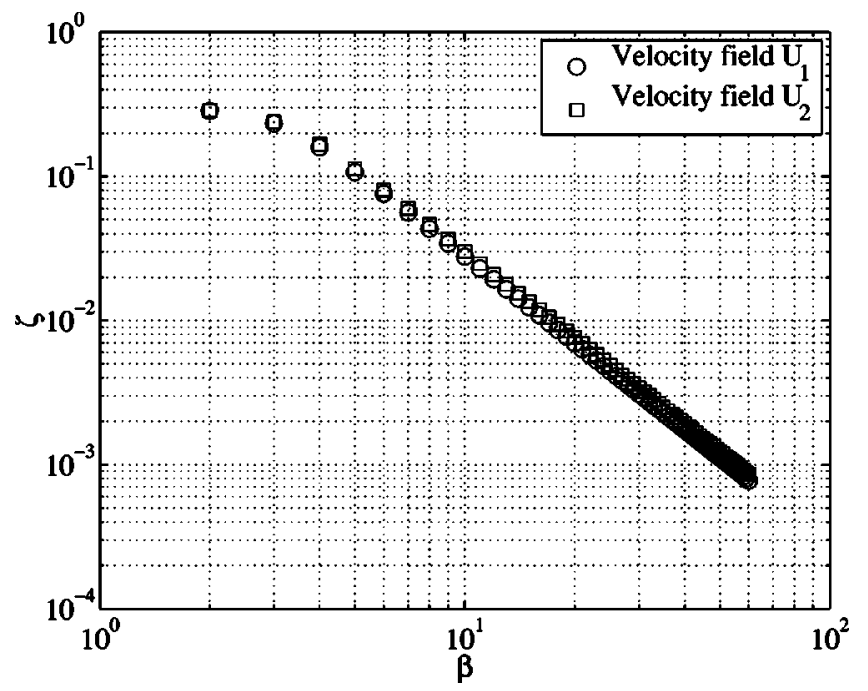

FIG. 1. Error parameter $\zeta$ [see definition in Eq. (69)] vs $\beta$ for the two flows $U_{1}$ [Eq. (48)] and $U_{2}$ [Eq. (50)].

Appendix A). This velocity field corresponds to a vortex with a nonzero circulation and a solid rotation core. Such velocity fields were obtained experimentally in water, to study the interaction of the vortex with surface waves [37]. In aeroacoustics, experimental vortices have smooth velocity profiles $[32,33]$ and the solid core vortex is a very poor approximation. In order to get closer to the experimental situation, we study polynomial velocity profiles:

$$
\begin{gathered}
U(r)=\sum_{i=1}^{i_{\max }} \gamma_{i}\left(\frac{r}{L_{m}}\right)^{i}, \quad r \leqslant L_{m}, \\
U(r)=\frac{\Gamma}{2 \pi r}, \quad r \geqslant L_{m},
\end{gathered}
$$

$\Gamma$ being the circulation of the mean flow,

$$
\Gamma=2 \pi L_{m} \sum_{i=1}^{i_{\max }} \gamma_{p}
$$

The mean-flow vorticity $\Omega$ confined inside the circle $r$ $=L_{m}$, has also a polynomial form

$$
\Omega=\frac{2 \gamma_{1}}{L_{m}}+\sum_{i=2}^{i_{\max }-1} \frac{(i+2)}{L_{m}} \gamma_{i}\left(\frac{r}{L_{m}}\right)^{i}, \quad r \leqslant L_{m}
$$

Then, choosing the $\gamma_{i}$, we can modelize a scattering problem by an axisymmetric vortex, including zero or nonzero circulation.

The outer solution remains the same, Eq. (14), whereas the inner solution (17) is expanded in series

$$
F_{n}^{i n}(r)=\sum_{p=0}^{\infty} \kappa_{p}^{n}\left(\frac{r}{L_{m}}\right)^{p}
$$

We show in Appendix C

$$
\begin{gathered}
\kappa_{p}^{n}=0 \text { for } p<|n|, \\
\kappa_{n}^{|n|}=1 \text { for } p=|n|, \\
\kappa_{p}^{n}=\Phi\left(\gamma_{1}, \ldots, \gamma_{\left.i_{\text {max }}, L_{m}, k\right)} \text { for } p>|n| .\right.
\end{gathered}
$$

The analytical expression of the function $\Phi$ is related to the mean-flow structure. The complete expression of the $\kappa_{p}^{n}$ depends on the mean-flow structure (70) and can be obtained, for a given $U$, by identifying order by order in $\left(r / L_{m}\right)$ terms of Eq. (16). We then get a recurrence relation between the $\kappa_{p}^{n}$.

As $L_{m}=L_{i}, b_{n} \equiv 0$ and $c_{n} \equiv 0$. Then, using Eqs. (20), we can express the sound wave from Eqs. (21) and (23).

\section{Number of terms in series}

For computational reasons, we realize the partial-wave summation on a finite number of terms, namely, $2 n_{\max }+1$ terms $\left(-n_{\max } \leqslant n \leqslant n_{\max }\right)$. The series in Eqs. (9) and (74) are simply convergent, so that the larger $\beta$ and $k r$ are, the bigger $n_{\max }$ must be.

The value of $n_{\max }$ has been discussed in scattering problems of electromagnetic waves by spherical conducting particles (Mie scattering) (see Ref. [53], Sec. 13.5). These problems, involving summations of spherical Bessel functions, are similar to our aeroacoustic one. From the analysis of the convergence behavior of the series and numerical computations of the series terms, Wiscombe [54] suggested a criterion for $n_{\max }$ :

$$
n_{\max }=x+4 x^{1 / 3}+2
$$

where in our case, $x=\beta$ for scattering amplitude computations [e.g., Eq. (31)] and $x=\max (\beta, k r)$ for sound field computations [e.g., Eq. (23)]. From our numerical computations, the above choice of $n_{\max }$ leads to a very good estimate of the sound quantities.

Also for computational reasons, we will denote $p_{\max }$ the number of terms in the series expansion (74) (for each fixed mode $\left.n,|n| \leqslant p \leqslant|n|+p_{\text {max }}\right)$. Of course, the larger $\beta$ and the polynomials' order (70) are, the larger the number of terms that should be kept in the series (74). We were not able to find a criterion analogous to Eq. (76) to quantify $p_{\max }$. From our computations with large $n_{\max }$, we find that $p_{\max }$ $=2 n_{\max }$ works fine.

\section{A zero-circulation vortical flow}

We consider in this section the scattering by a vortical flow with no circulation. This type of flow without circulation has a great theoretical interest: it is possible to perform the analysis with usual sound scattering methods because no spurious divergencies appear in the scattered quantities $[19,20]$. Moreover, this type of mean flow has a great importance in experimental situations such as von Kármán street [55-57] or normal modes of surface waves [47]. 

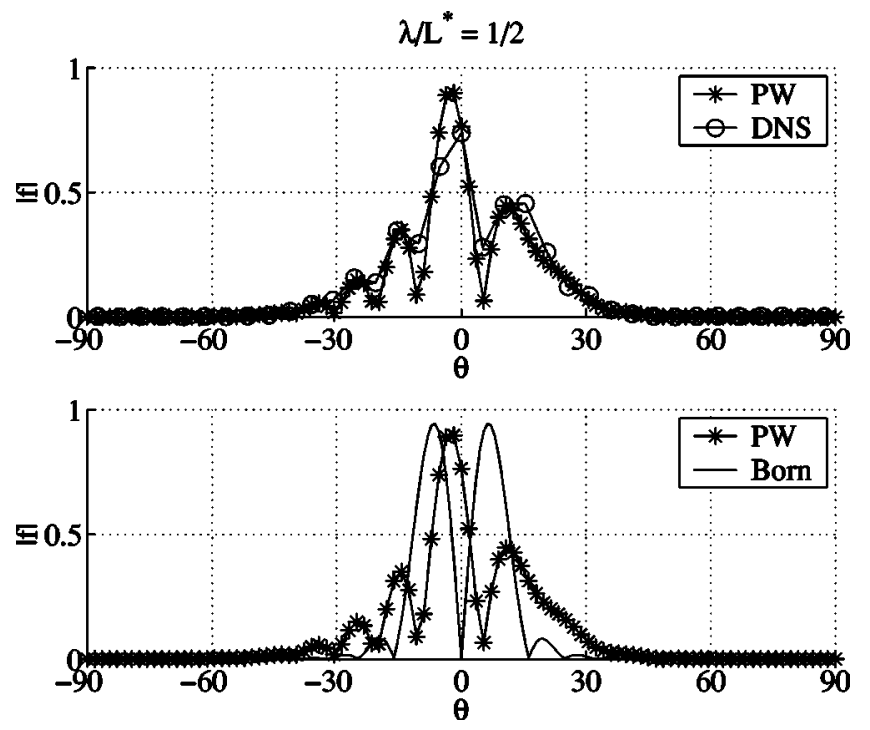

FIG. 2. Scattering amplitudes $|f|$, resulting from the interaction of a plane sound wave $\left(\lambda / L^{*}=0.5\right)$ with the mean flow $U_{2}$ [Eq. (50)] with $\mathcal{M} \simeq 0.1$, with respect to the scattering angle $\theta$ (in degrees), partial-wave computations; o, direct numerical simulation; - , first Born approximation.

We analyze the scattered sound wave from the zerocirculation mean flow (50). From Eqs. (21)-(23), we can compute the total sound field $\rho_{s}$, its scattered part $\rho_{\text {scatt }}$, and the scattering amplitude $f(\theta)$. We can then compare the results from the PW method with results from the first Born approximation [19]. As said before, the Born approximation is valid if the flow satisfies $\mathcal{M} \beta=\mathcal{M} 2 \pi L_{m} / \lambda \ll 1$, whereas the PW method requires $\beta \gg 1$. We also use a direct numerical simulation (DNS) of the sound-mean-flow interaction $[58,59]$ to analyze the scattering behavior. This DNS allows
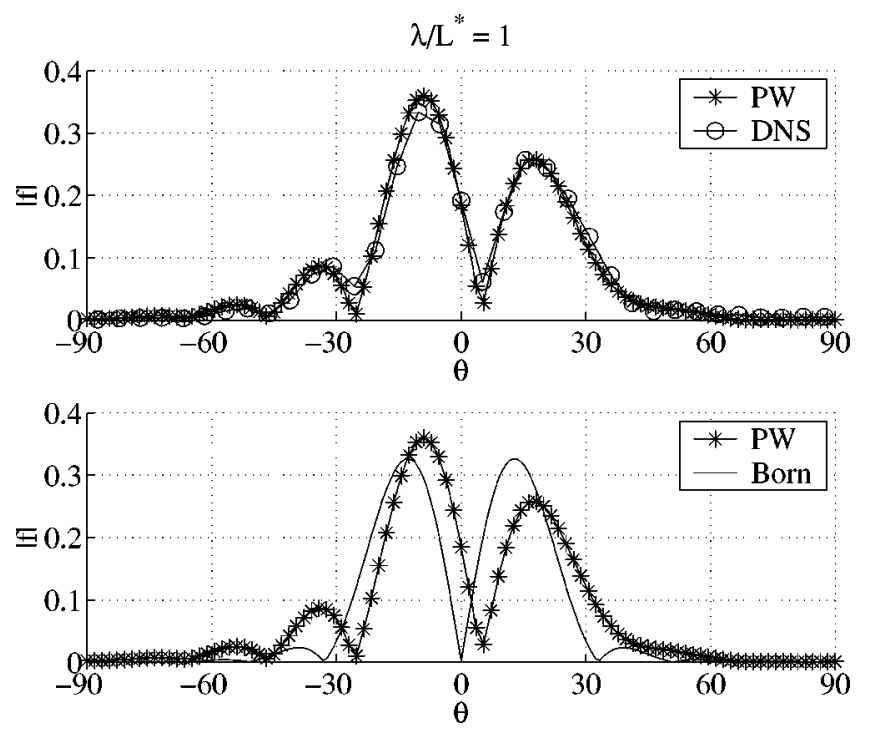

FIG. 3. Scattering amplitudes $|f|$, resulting from the interaction of a plane sound wave $\left(\lambda / L^{*}=1\right)$ with the mean flow $U_{2}$ [Eq. (50)] with $\mathcal{M} \simeq 0.1$, with respect to the scattering angle $\theta$ (in degrees). *, partial-wave computations; o, direct numerical simulation; - , first Born approximation.
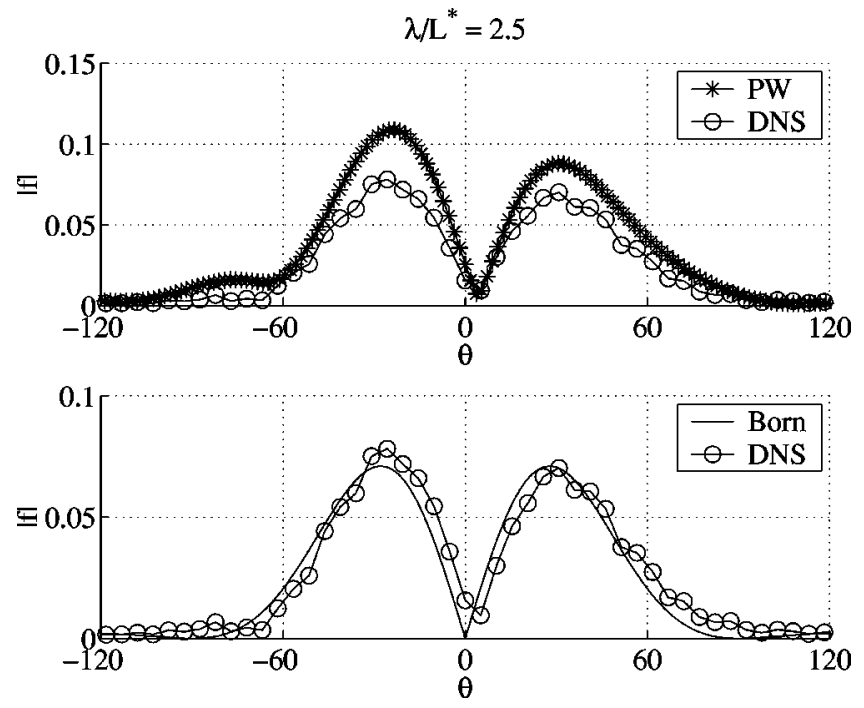

FIG. 4. Scattering amplitudes $|f|$, resulting from the interaction of a plane sound wave $\left(\lambda / L^{*}=2.5\right)$ with the mean flow $U_{2}$ [Eq. (50)] with $\mathcal{M} \simeq 0.1$, with respect to the scattering angle $\theta$ (in degrees). *, partial-wave computations; o, direct numerical simulation; - , first Born approximation.

us to study the interaction for a large range of $\lambda / L^{*}$ ratios, where $L^{*}$ is the vortex core radius (location of the velocity maximum).

We present in Figs. 2-5 the scattering amplitude $f$ for the mean flow $(50) \quad\left(\mathcal{M} \simeq 0.1\right.$, largest velocity for $L^{*}$ $=0.01 \mathrm{~m}$ ), computed from the three methods, for different $\lambda / L^{*}$ ratios. The agreement is good between the DNS and one of the two theoretical methods, without any free parameters. As expected, the Born approximation falls down for $\lambda / L^{*} \leqslant 2.5(\mathcal{M} \beta \geqslant 0.5)$, whereas the PW computations are valid for $\lambda / L^{*} \leqslant 1 \quad(\beta \geqslant 12)$.
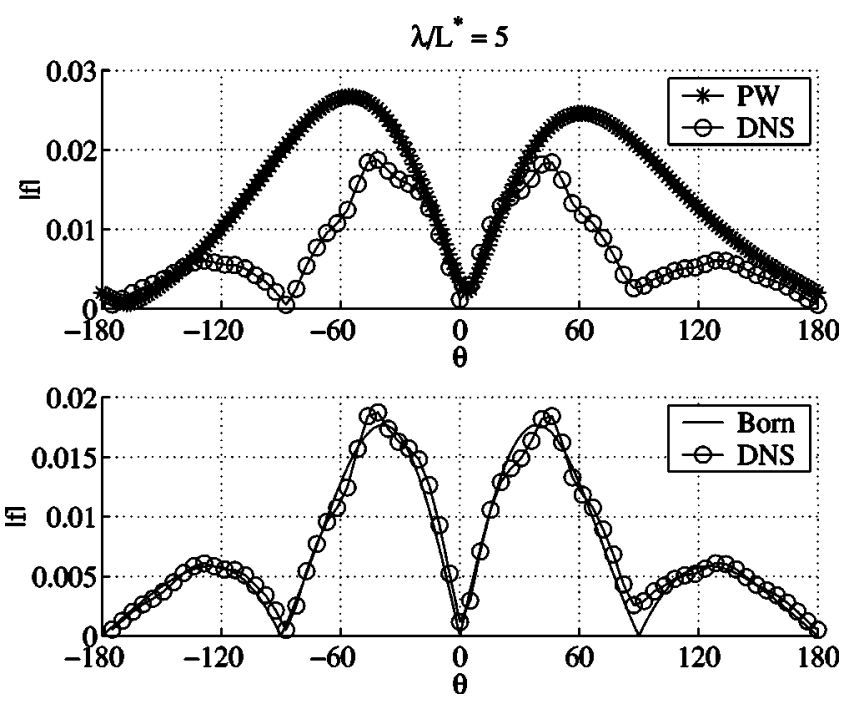

FIG. 5. Scattering amplitudes $|f|$, resulting from the interaction of a plane sound wave $\left(\lambda / L^{*}=5\right)$ with the mean flow $U_{2}$ [Eq. (50)] with $\mathcal{M} \simeq 0.1$, with respect to the scattering angle $\theta$ (in degrees). *, partial-wave computations; o, direct numerical simulation; - , first Born approximation. 

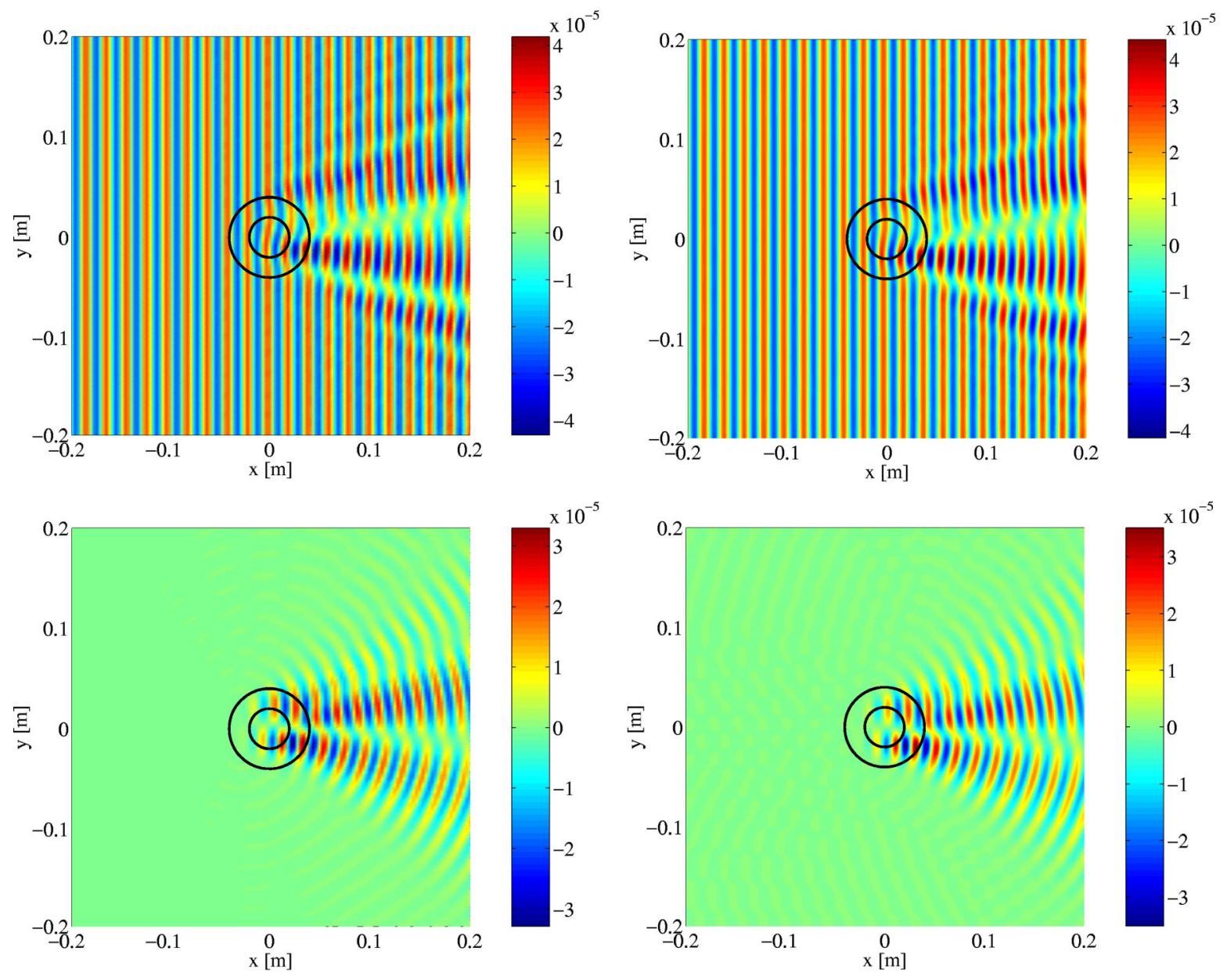

FIG. 6. Sound field resulting from the interaction of an incident plane monochromatic sound wave (propagating from the left to the right of the domain, $\lambda / L^{*}=1$ ) with the mean flow $U_{2}$ [Eq. (50)], $\mathcal{M} \simeq 0.1 . \beta \simeq 12$. Left-upper, sound wave $\rho_{s}$ from the partial-wave computations; right-upper, sound wave $\rho_{s}$ from the direct numerical simulation. Left-lower, scattered sound wave $\rho_{\text {scat }}$ from the partial-wave computations; right-lower, scattered sound wave $\rho_{\text {scat }}$ from the direct numerical simulation. Circles locate the position of the two opposite sign vorticity domains. Units are in meters on each axis.

We have also computed the total sound field $\rho_{s}$ and its scattered part [60] $\rho_{\text {scat }}$ with the PW method and the DNS. We use a square domain of size $20 L^{*} \times 20 L^{*}$. The fluid parameters are those of air under normal conditions. The incident plane wave propagates from the left to the right of the domain and we take $\rho_{s 0}=2.63 \times 10^{-5} \mathrm{~m}^{3} \mathrm{~kg}^{-1} \approx 2.6$ $\times 10^{-5} \rho_{\text {air }}$ to ensure linear sound waves. Results are presented in Fig. 6 for $\lambda / L^{*}=1(\beta \simeq 12)$ and on Fig. 7 for $\lambda / L^{*}=1 / 2 \quad(\beta \simeq 25)$ where the PW method is valid. The agreement is also rather good between the two methods, without any free parameters.

In this range of wavelengths, the scattering process is more efficient as the wavelength decreases. Moreover, the wave front perturbation, present just after the sound-vortex crossing, decreases as the sound wave goes away from the vortex core. This observation is consistent with the physical situation: as no topological default is present, this perturbation disappears at large distances from the vortex, and is not to be confused with the real dislocation observed when the vortical flow has a finite circulation.

\section{A finite circulation vortical flow}

This type of flow was extensively studied in experimental situations with surface waves and sound waves. A solid rotation core well modelizes some experimental configurations $[31,37]$ but a more general velocity profile is needed to take into account general experimental setups [32,33]. These kind of vortices have also a theoretical importance: they create a dislocation of the wave front, and it is possible to investigate in detail the phase-shift process and to perform in a quantitative way the Berry's analogy $[33,34,38]$.

The simplest polynomial expansion (70), which gives a continuous vorticity, decreasing to 0 with a zero slope at $r$ $=L_{m}$, is $U_{3}$, with

$$
\begin{gathered}
U_{3}(r)=\frac{\omega_{3} r}{2}\left(1-\frac{3 r^{2}}{2 L_{m}^{2}}+\frac{4 r^{3}}{5 L_{m}^{3}}\right), r \leqslant L_{m}, \\
U_{3}(r)=\frac{\Gamma_{3}}{2 \pi r}, \quad r \geqslant L_{m},
\end{gathered}
$$



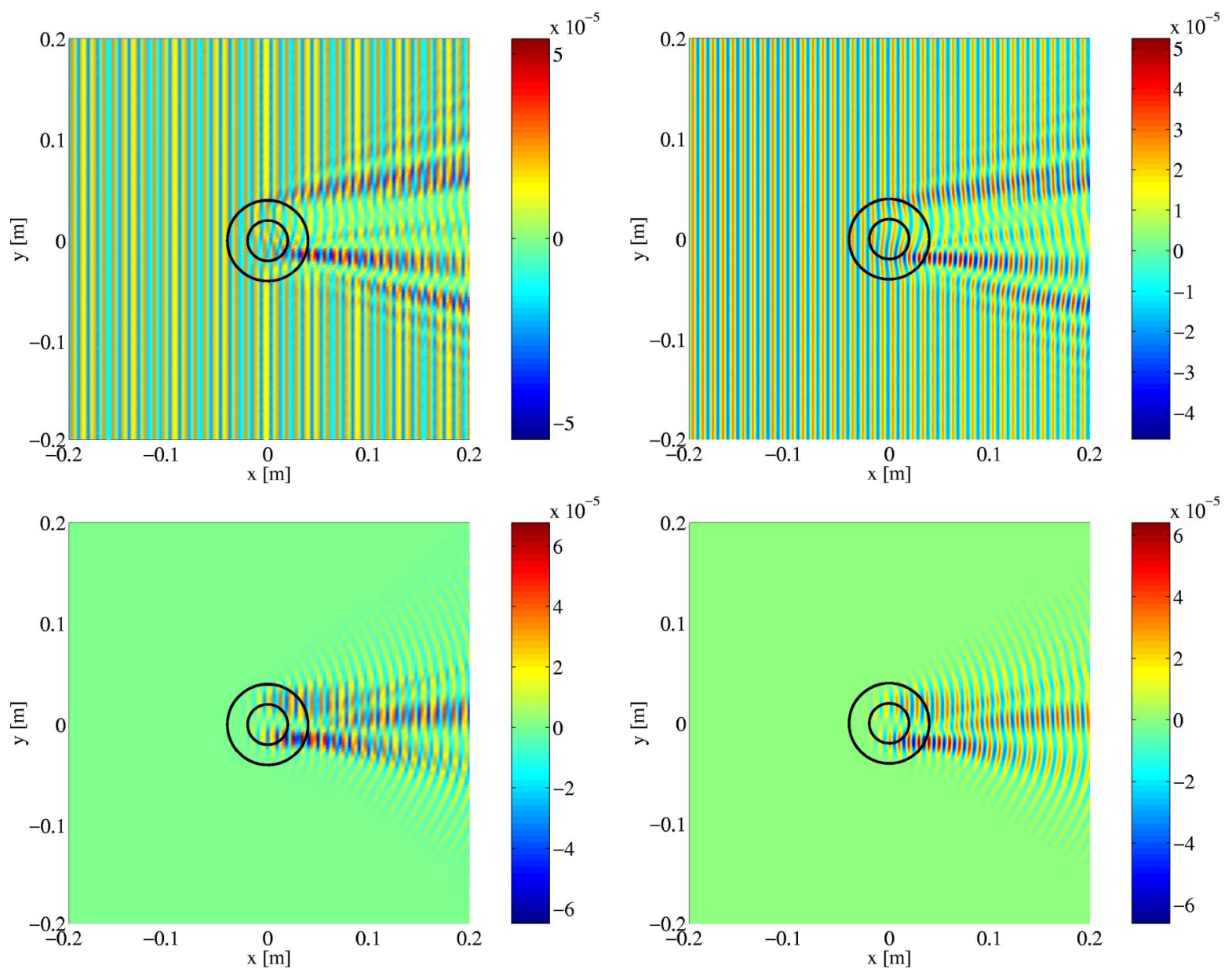

FIG. 7. Sound field resulting from the interaction of an incident plane monochromatic sound wave (propagating from the left to the right of the domain, $\lambda / L^{*}=0.5$ ) with the mean flow $U_{2}$ [Eq. (50)], $\mathcal{M} \simeq 0.1$. $\beta \simeq 25$. Left-upper, sound wave $\rho_{s}$ from partial-wave computations; right-upper, sound wave $\rho_{s}$ from direct numerical simulation. Left-lower,xs scattered sound wave $\rho_{\text {scat }}$ from the partial-wave computations; right-lower, scattered sound wave $\rho_{\text {scat }}$ from the direct numerical simulation. Circles locate the position of the two opposite sign vorticity domains. Units are in meters on each axis.

$U_{3}$ is maximum at $r=L^{*} \simeq 0.64 L_{m}$. The circulation is $\Gamma_{3}$ $=3 \pi \omega_{3} L_{m}^{2} / 10$, ensuring continuity of the velocity at $r$ $=L_{m}$. The vorticity $\Omega_{3}$ reads

$$
\begin{gathered}
\Omega_{3}(r)=\omega_{3}\left(1-\frac{3 r^{2}}{L_{m}^{2}}+\frac{2 r^{3}}{L_{m}^{3}}\right), \quad r \leqslant L_{m}, \\
\Omega_{3}(r)=0, \quad r \geqslant L_{m} .
\end{gathered}
$$

This velocity field is a solution of the ideal fluid equations and is smooth enough to mimic experimental profiles. The detailed calculations of the inner solution (74) are given in Appendix C.

We present results from the interaction of a plane sound wave with the mean flow $(77)\left(\mathcal{M} \simeq 3.9 \times 10^{-2}\right.$, largest velocity for $\left.L^{*}=0.01 \mathrm{~m}\right)$ for $\lambda / L^{*} \simeq 1 \quad(\beta \simeq 9.8$, Fig. 8), $\lambda / L^{*} \simeq 3 / 4$ ( $\beta \simeq 13.1$, Fig. 9 , left side $)$, and $\lambda / L^{*} \simeq 0.625$ ( $\beta \simeq 15.8$, Fig. 9 , right side). Computations were performed with a domain of size $0.4 \mathrm{~m} \times 0.4 \mathrm{~m}\left(\simeq 26 L_{m}\right)$ and the incident sound wave propagates from the left to the right of the domain. In each case, the fluid parameters are those of air under normal conditions, and we took $\rho_{s 0}=2.6 \times 10^{-5} \rho_{\text {air }}$ to ensure linear sound waves. Again the agreement between our PW method and the DNS is rather satisfactory (Fig. 8).

For these choices of $\lambda / L^{*}$ ratios, the scattering is more efficient as the wavelength decreases. Moreover, we can observe a dislocation after the sound-vortex interaction, which seems to be of constant amplitude (compare to the zero circulation case shown in Figs. 6 and 7). This dislocation, with amplitude and orientation depending on the parameter $\alpha$-that is the intensity of the mean flow and the orientation of the vortex rotation with regard to the incident wave direction of propagation - is the signature of the Aharonov-Bohm effect. These results are very similar to the results of Vivanco et al. [37] computed for surface waves scattered by a vortex in solid rotation. This is obvious, since the dislocation depends on the vortex circulation only, which quantifies the topological defect in the velocity field, and not on the core structure.

Another prominent feature of the interaction of a sound wave and a vortex with circulation is the dissymmetry of the scattered wave (compare Figs. 6 and 7 with Figs. 8 and 9). It 

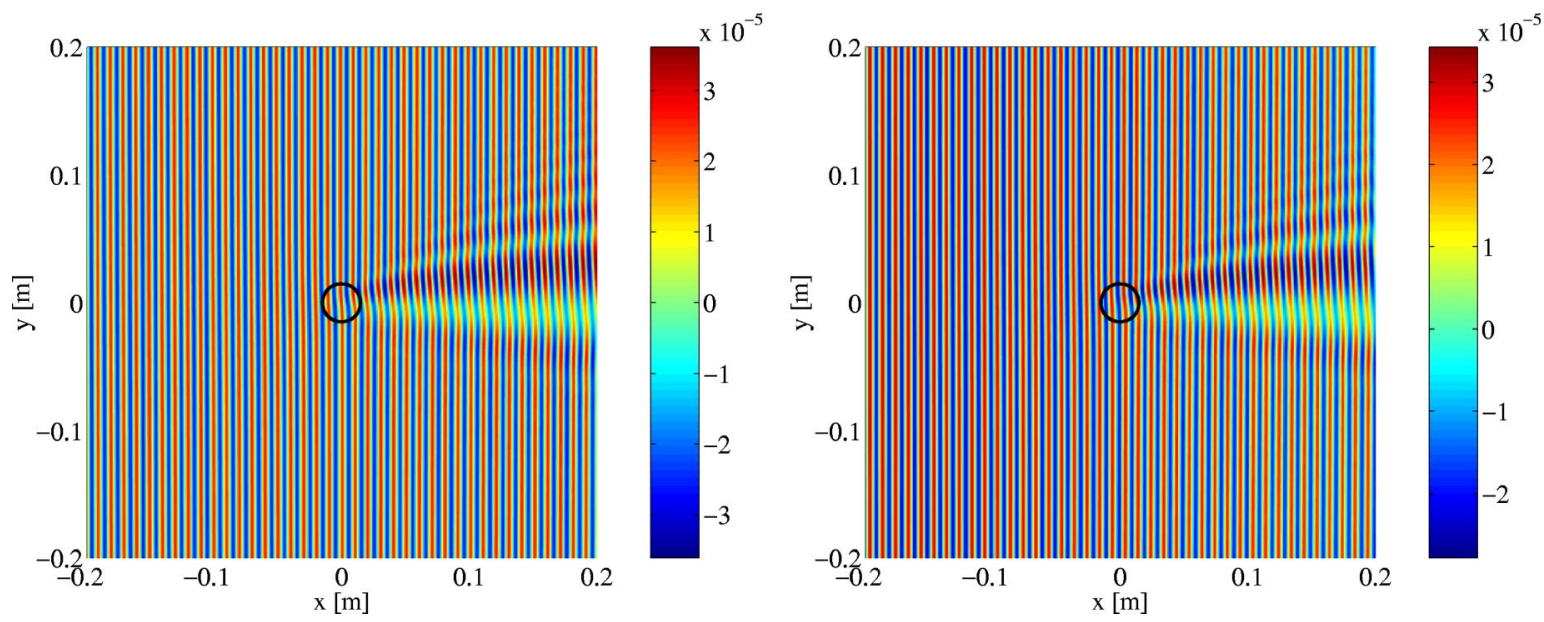

FIG. 8. Sound wave $\rho_{s}$ resulting from the interaction of an incident plane monochromatic sound wave (propagating from the left to the right of the domain, $\lambda / L^{*}=1$ ) with the mean flow $U_{3}$ [Eq. (77)], $\mathcal{M} \simeq 0.039$. $\beta \simeq 9.8$. Left, partial-wave computations; right, direct numerical simulation. Circle locates the position of the vortex core (rotating counterclockwise). Units are in meters on each axis.

is crucial, in order to exhibit this property that is observed experimentally $[37,47]$, to calculate the sound field inside the vortex core.

Following previous works [28,31,33,34,37], we want to emphasize here the importance of fluid dynamics experiments (acoustic or surface waves) for the study of the Aharonov-Bohm effect: we are able to analyze both the amplitude and phase of the wave [38]. The PW computations give access to the full structure of the complex sound field [see Eqs. (21)-(23)]. Thus, writing

$$
\rho_{s}=\left|\rho_{s}\right| e^{-2 i \pi \nu t} e^{i \phi},
$$

we can easily extract the amplitude $\left|\rho_{s}\right|$ and the phase shift $\phi$ of the wave with respect to the incident wave.

Figure 10 shows cross sections of the amplitude $\left|\rho_{s}\right|$ for $x=0.19 \mathrm{~m}$ for different $\lambda / L^{*}$ ratios. We also present in Fig. 11 cross sections of the phase shift $\phi$ for $x=0.19 \mathrm{~m}$ for different $\lambda / L^{*}$ ratios. Far from the $y=0$ plane, the sound wave is undisturbed by the mean flow: the phase is almost constant and the sound amplitude is equal to 1 . It means that scattering effects are very small in these regions. The analysis of the amplitude behavior in these regions does not give informations about the sound wave propagation through the vortex flow. Nevertheless, the sound-vortex interaction can be detected by measuring the phase jump $\Delta \phi$ from one side to the other. Following Ref. [34], this phase jump can be expressed as

$$
\Delta \phi=\frac{2 \pi \Gamma}{\lambda c_{0}} .
$$

Plotting $\Delta \phi$ with respect to $1 / \lambda$ gives informations about the flow: we can get the flow circulation $\Gamma$ from the curve slope (Fig. 12). Practically, from the cross sections of the phase, it is not easy to define $\Delta \phi$ because interference patterns between the incident wave, the dislocated wave, and the scattered wave are superimposed on the dislocation pattern producing the phase jump. We decided to define $\Delta \phi$ between
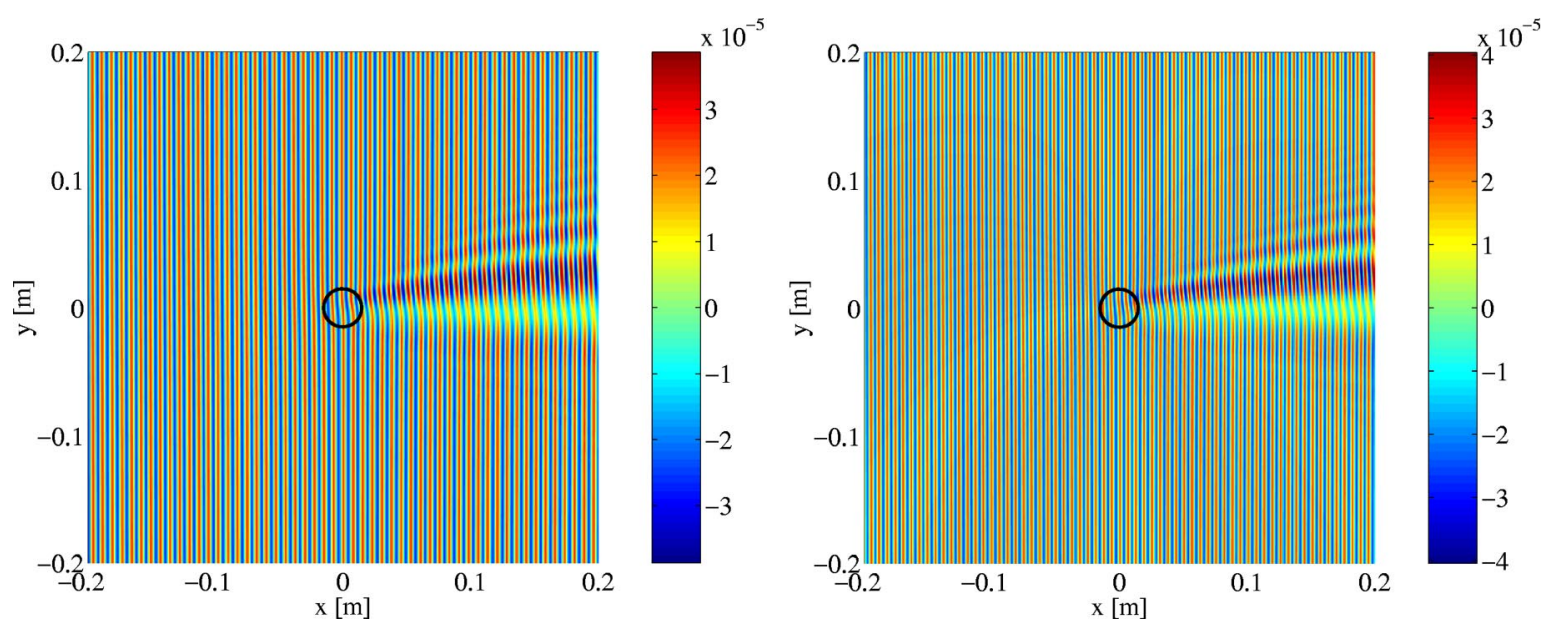

FIG. 9. Sound wave $\rho_{s}$ resulting from the interaction of an incident plane monochromatic sound wave (propagating from the left to the right of the domain) with the mean flow $U_{3}$ [Eq. (77)], $\mathcal{M} \simeq 0.039$. Partial-wave computations with left, $\lambda / L^{*}=3 / 4, \beta \simeq 13.1 ;$ right, $\lambda / L^{*}=0.625, \beta \simeq 15.8$. Circle locates the position of the vortex core (rotating counterclockwise). Units are in meters on each axis. 


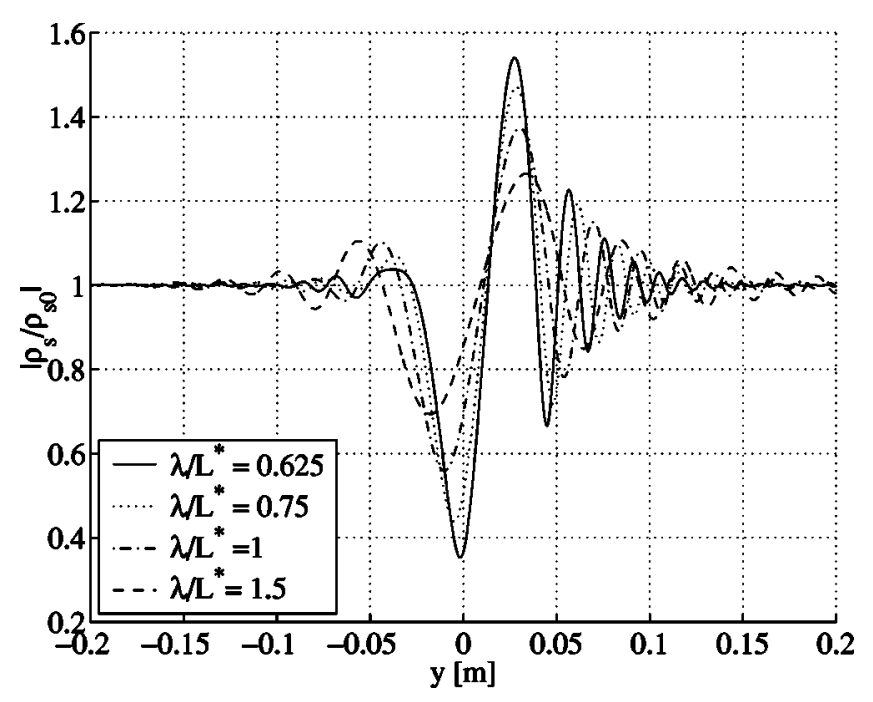

FIG. 10. Cross sections, for $x=0.19 \mathrm{~m}$, of the amplitude $\left|\rho_{s}\right|$, for different $\lambda / L^{*}$ ratios.

the minimum and the maximum values of the phase shift (see Fig. 11), which is obviously an overestimate. In so doing, we get $\Gamma \simeq 1.26 \mathrm{~m}^{2} \mathrm{~s}^{-1}$, to be compared with the chosen value from the mean-flow $(77)$ properties $\left(\mathcal{M} \simeq 3.9 \times 10^{-2}\right.$, largest velocity for $\left.L^{*}=0.01 \mathrm{~m}\right): \Gamma^{i m} \simeq 1.16 \mathrm{~m}^{2} \mathrm{~s}^{-1}$. The above choice for the phase jump amplitude gives a good value of the vortical-flow circulation.

\section{CONCLUSION}

We have described a semianalytical method to analyze sound-vortical-flow-interactions in two-dimensional cases. This method is based on a partial-wave expansion (an angular Fourier transform) of a modified wave equation and is valid in the short wavelength limit for small Mach number flows. It is more powerful than ray-tracing methods because it gives, without extra computations, the sound wave amplitude and phase in the whole interaction domain, and because it is less restrictive on the smallness of the wavelength. It

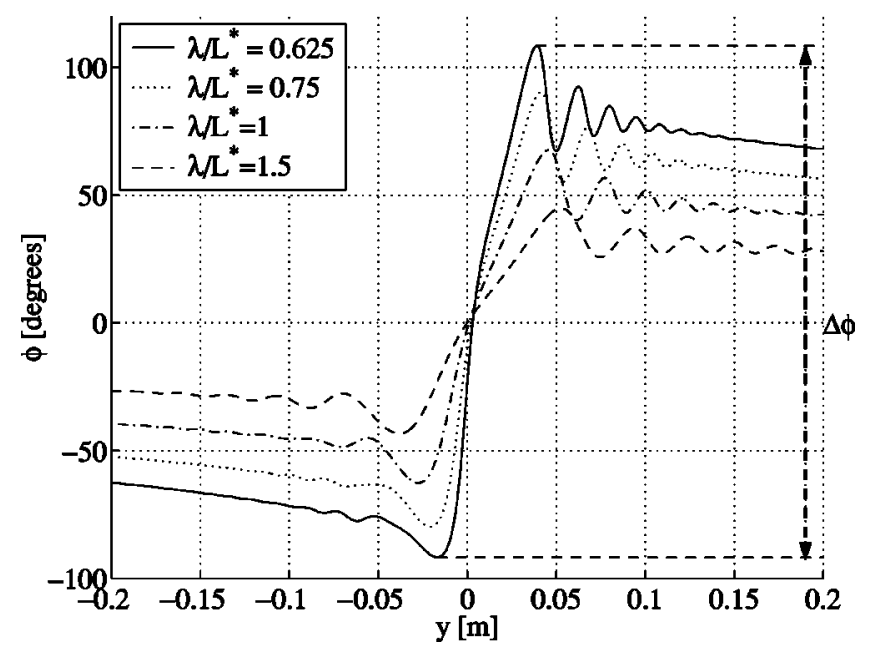

FIG. 11. Cross sections, for $x=0.19 \mathrm{~m}$, of the phase shift $\phi$ (in degrees), for different $\lambda / L^{*}$ ratios.

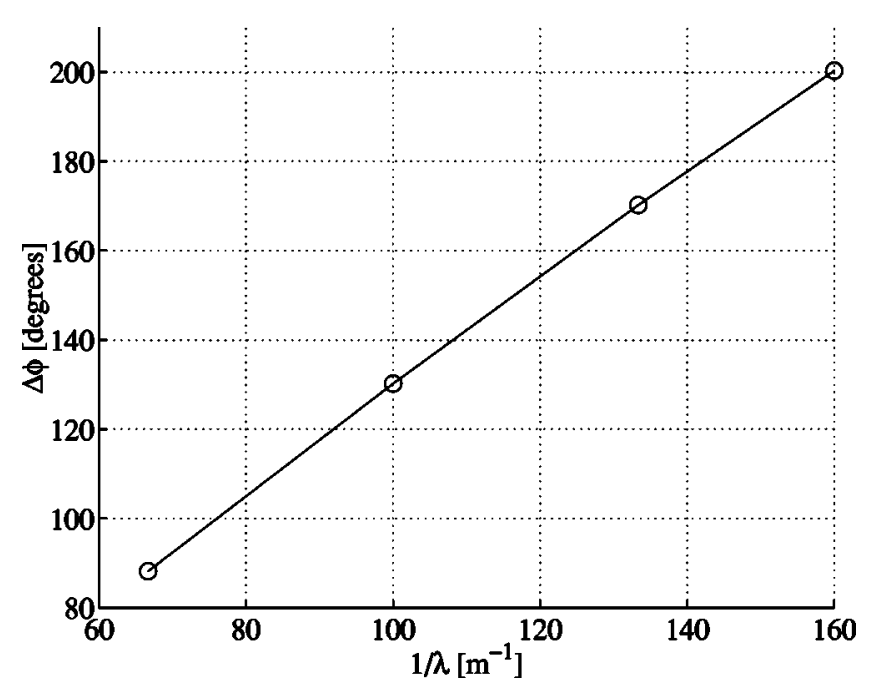

FIG. 12. Phase jump $\Delta \phi$ (in degrees) with respect to $1 / \lambda$ (in $\left.\mathrm{m}^{-1}\right)$.

also gives a better description than Born approximation because we are not restricted to the scattered part of the wave (the far field), and because vortical flows with finite circulation are amenable to our analysis.

We analyzed in detail the range of validity of our method: the method is valid for $(2 \pi L / \lambda) \geqslant 12-15$. Moreover, we showed that in this short wavelength limit, the partial wave method preserves the acoustical energy conservation. We also gave some examples of sound scattering by vortical flows and we analyzed the behavior of the sound fields. We extend previous calculations to the case of smooth vorticity profiles that are essential to take into account actual experimental aeroacoustics problems.

Although approximate, our method is essentially nonperturbative and allows a satisfactory description of wave front dislocation by a finite circulation vortex, which corresponds to a topologically singular velocity field. Zero-circulation vortices are not topological defects, and the wave front perturbation that appears near the vortex core decreases rapidly with dicreasing distance.

Those sound-mean-flow interaction problems are formally close to quantum scattering problems. The most important change is that sound waves always penetrate into the vortical flow. A nontrivial physical consequence is the great dissymmetry of the scattering pattern with finite circulation vortices. A large number of papers in quantum mechanics deal with scattering by various potentials, but only a few of them take into account the internal structure of the scatterers.

A generalization of our method to more complicated flows (e.g., involving more than one vortex, three-dimensional problems) seems to be an hard task and remains a open problem in order to analyze multiple scattering events in these flows. One way of solving the problem of two vortices could be to introduce elliptic coordinates and Mathieu functions [61].

\section{ACKNOWLEDGMENTS}

We are grateful to S. Fauve and F. Lund for helpful discussions. R.B. also thanks A. Folacci for fruitful suggestions. 
We gratefully acknowledge support from contract ECOSCONICYT.

\section{APPENDIX A: SOUND SCATTERING BY A SOLID ROTATION VORTEX}

Let us consider the vortical flow produced by a vortex with a core in solid rotation:

$$
\begin{gathered}
U(r)=\omega_{0} r / 2, \quad r \leqslant L_{m}, \\
U(r)=\frac{\Gamma}{2 \pi r}, \quad r \geqslant L_{m},
\end{gathered}
$$

with $\Gamma=\pi \omega_{0} L_{m}^{2}$ in order to ensure continuity of the velocity. This vortical flow was considered by Coste and coworkers in surface wave scattering by vortex flows $[28,37]$ and also by Roux et al. [31] to analyze the acoustical Aharonov-Bohm effect.

The outer solution is given by Eq. (14) whereas the inner solution (17) can be expressed in terms of the Bessel function $J$ :

$$
F_{n}^{i n}(r)=J_{|n|}\left(k_{n} r\right)
$$

with

$$
k_{n}=k-\frac{n \omega_{0}}{2 c_{0}}
$$

We have kept the only solution that is finite at $r=0$.

If $2 c_{0} k / \omega_{0}$ is an integer $n_{0}, k_{n}$ vanishes for $n=n_{0}$. In this special case, the inner solution can be found by assuming $F_{n_{0}}^{i n}(r) \propto r^{p}$. This leads to $p= \pm n_{0}$ and we choose the solution $p=n_{0}$ to get a bounded solution at the origin:

$$
F_{n_{0}}^{\text {in }}(r)=r^{n_{0}} .
$$

As $L_{i}=L_{m}, b_{n} \equiv 0$ and $c_{n} \equiv 0$. The complete solution can be achieved by solving the system (20).

\section{APPENDIX B: BORN APPROXIMATION}

Starting from the basic equations (3) and (4) describing the interaction of a sound wave (sound pulsation $2 \pi \nu$ ) with a two-dimensional stationary mean flow, one can derive, in the Born approximation, a formal expression for the scattered amplitude (26) [19]:

$$
f(\theta)=\frac{1}{2 c_{0}} \sqrt{\frac{i 2}{c_{0}}} \cos (\theta) \cot (\theta / 2) \widetilde{\Omega}_{z}\left(\vec{k}_{R}-\vec{k}_{i}\right)
$$

with $\vec{k}_{i}=2 \pi \nu_{0} / c_{0} \hat{\imath}$ the wave vector of the incident plane wave that propagates in direction of the unit vector $\hat{\imath}, \vec{k}_{R}$ $=2 \pi \nu / c_{0} \hat{R}$ is the wave vector in the direction of observation $\hat{R}$, and

$$
\widetilde{\Omega}(\vec{q})=\frac{1}{(2 \pi)^{2}} \int \vec{\Omega}(\vec{r}) e^{-i \vec{q} \cdot \vec{r}} d^{2} \vec{r}
$$

is the spatial Fourier transform of the vorticity field. This expression allows to use sound as a probe of turbulent flows $[20,62,63]$.
In the forward direction $\theta \simeq 0$, one can easily see that $f$ diverges for a nonzero-circulation mean flow. This is a wellknown behavior in the Born approximation $[30,46]$. This traces back to the fact that the physical effect of a nonzero circulation is the dislocation of the incident wave front. In the forward direction, the incident wave undergoes a modification of the same amplitude as itself. This is a nonperturbative effect, inaccessible to a perturbative approach such as the Born expansion.

For axisymmetric flows in two dimensions, taking $[\mathrm{Oz}$ ) as the symmetry axis, $\Omega=\Omega(r) \hat{z}$ in cylindrical coordinates and the Fourier transform (B2) reduces to

$$
\widetilde{\Omega}(\vec{q})=2 \pi \int_{0}^{\infty} \Omega(r) J_{0}(q r) r d r .
$$

\section{APPENDIX C: COMPUTATION OF THE INNER SOLUTION SERIES}

We must solve Eq. (16),

$$
\rho_{n}^{\prime \prime}+\frac{\rho_{n}^{\prime}}{r}+\left[k^{2}-\frac{2 n k}{c_{0} r} \sum_{i=1}^{i_{\max }} \gamma_{i}\left(\frac{r}{L_{m}}\right)^{i}-\frac{n^{2}}{r^{2}}\right] \rho_{n}=0 .
$$

For each value of $n$, we introduce the index $p_{0} \geqslant 0$ of the first nonzero term of Eq. (74):

$$
\rho_{n}=\sum_{p=p_{0}}^{\infty} \kappa_{p}^{n}\left(\frac{r}{L_{m}}\right)^{p}
$$

Injecting this in Eq. (C1), we then determine the constants $\kappa_{p}^{n}$ with the requirement that the coefficient of each power of $r / L_{m}$ be zero.

When $r \rightarrow 0$, the leading order term is $\left(r / L_{m}\right)^{p_{0}-2}$, with the coefficient $\left(p_{0}^{2}-n^{2}\right) \kappa_{p_{0}}^{n} / L_{m}^{2}$. We thus get the general result

$$
\kappa_{p_{0}}^{n}=0 \text { for } p_{0} \neq|n|
$$

or

$$
\kappa_{p_{0}}^{n} \neq 0 \quad \text { for } p_{0}=|n|
$$

Thus, for a given mode $\rho_{n}$, the series expansion starts at $p$ $=p_{0}=|n|$. We take the positive value of $p_{0}$ in order to get a finite acoustical field at $r=0$. In this general calculation, we recover the asymptotic behavior near the origin of the analytical solutions (55), (62), and (A2). In this respect, the velocity fields (48), (50), and (A1) are only special cases of the polynomial ones considered in this appendix.

The coefficients $\kappa_{p}^{n}$ for $p>|n|$ are then calculated by recurrence. The general expression, for the velocity field (70), is too cumbersome and can be expressed as

$$
\begin{gathered}
\kappa_{p}^{n}=0 \text { for } p<|n|, \\
\kappa_{n}^{|n|}=1 \text { for } p=|n| \\
\kappa_{p}^{n}=\Phi\left(\gamma_{1}, \ldots, \gamma_{i_{\text {max }}}, L_{m}, k\right) \text { for } p>|n| .
\end{gathered}
$$


The analytical expression of the function $\Phi$ depends on the mean-flow structure.

We will now focus on the particular case (77). A straightforward calculation gives the recurrence relations for the coefficients $\kappa_{p}^{n}$. The particular cases are

$$
\begin{gathered}
\kappa_{p}^{n}=0 \quad(p<|n|), \\
\kappa_{|n|}^{n} \neq 0, \\
\kappa_{|n|+1}^{n}=0 \\
\frac{4(|n|+1)}{L_{m}^{2}} \kappa_{|n|+2}^{n}+\left(k^{2}-\frac{n k \omega_{3}}{c_{0}}\right) \kappa_{|n|}^{n}=0 \\
\frac{8(|n|+2)}{L_{m}^{2}} \kappa_{|n|+3}^{n}=0, \\
\left.\frac{5(2|n|+5)}{L_{m}^{2}} \kappa_{|n|+5}^{n}-\frac{n}{5} \frac{n k \omega_{3}}{c_{0}}\right) \kappa_{|n|+2}^{n}+\frac{3}{2} \frac{n k \omega_{3}}{c_{0}} \kappa_{|n|}^{n}=0 \\
k_{|n|}^{n}=0
\end{gathered}
$$

[1] I.G. Jonsson, in Wave-Current Interactions, edited by B. Le Mehaute and M. H. Hanes, Ocean Engineering Science, The Sea Vol. 9A (Wiley, New York, 1990), pp. 65-120.

[2] L.M.B.C. Campos, Rev. Mod. Phys. 58, 117 (1986).

[3] A.M. Obukhov, Waves Random Media 4, 9 (1994).

[4] D. Sheres and K.E. Kenyon, Int. J. Remote Sens. 11, 27 (1990).

[5] M. Karweit, P. Blanc-Benon, D. Juvé, and G. Comte-Bellot, J. Acoust. Soc. Am. 89, 52 (1991).

[6] V.E. Ostashev, V. Mellert, R. Wandand, and F. Gerdes, J. Acoust. Soc. Am. 102, 2561 (1997).

[7] A. Ishimaru, Wave Propagation and Scattering in Random Media (Academic Press, New York, 1978).

[8] V.A. Polyanskaya, Akust. Zh. 31, 698 (1985) [Sov. Phys. Acoust. 31, 376 (1985)].

[9] D. Blokhintzev, J. Acoust. Soc. Am. 18, 322 (1946).

[10] E.T. Kornhauser, J. Acoust. Soc. Am. 25, 945 (1953).

[11] T.M. Georges, J. Acoust. Soc. Am. 51, 206 (1972).

[12] E.G. Broadbent, J. Inst. Math. Appl. 19, 1 (1977).

[13] J. Lighthill, ICASE Report No. 92-52, 1992 (unpublished).

[14] D.S. Jones, Philos. Trans. R. Soc. London, Ser. A 255, 341 (1963).

[15] T. Colonius, S.K. Lele, and P. Moin, J. Fluid Mech. 260, 271 (1994).

[16] S.M. Candel, J. Fluid Mech. 83, 465 (1977).

[17] M. Mathiesen, J. Geophys. Res. 92, 3905 (1987).

[18] R.H. Kraichnan, J. Acoust. Soc. Am. 25, 1096 (1953).

[19] A.L. Fabrikant, Akust. Zh. 29, 262 (1983) [Sov. Phys. Acoust. 29, 152 (1983)].

[20] F. Lund and C. Rojas, Physica D 37, 508 (1989).

[21] A.L. Fetter, Phys. Rev. 136, 1488 (1964). whereas the general term, for $p>5$, is given by

$$
\begin{gathered}
\frac{p(2|n|+p)}{L_{m}^{2}} \kappa_{|n|+p}^{n}+\left(k^{2}-\frac{n k \omega_{3}}{c_{0}}\right) \kappa_{|n|+p-2}^{n} \\
\quad+\frac{n k \omega_{3}}{c_{0}}\left(\frac{3}{2} \kappa_{|n|+p-4}^{n}-\frac{4}{5} \kappa_{|n|+p-5}^{n}\right)=0
\end{gathered}
$$

Obviously, all $\kappa_{p}^{n}$ are proportional to $\kappa_{|n|}^{n}$, which is not specified in this calculation. According to the general solution (17), we write $\kappa_{|n|}^{n} \equiv 1$, with the proportionnality constant now denoted by $a_{n}$.

Even in this simple case, we are unable to get an explicit expression for the function $\Phi$ of Eq. (75). However, the system (C5) with (C6), together with the symbolic computation capabilities of MATHEMATICA are sufficient to calculate $\rho_{n}$ defined in Eq. (C2) up to any desirable order. In the particular cases of the velocity potentials (48), (50), and (A1), it is a straightforward exercise to verify that Eq. (C5) and (C6) give the well-known series expansions of the analytic solutions (55), (62), and (A2).
[22] J. Reinschke, W. Möhring, and F. Obermeier, J. Fluid Mech. 333, 273 (1997).

[23] M. Umeki and F. Lund, Fluid Dyn. Res. 21, 201 (1997).

[24] G.M. Golemshtok and A.L. Fabrikant, Akust. Zh. 26, 383 (1980) [Sov. Phys. Acoust. 26, 209 (1980)].

[25] A.M. Obukhov, C. R. (Dokl.) Acad. Sci. URSS 30, 616 (1941).

[26] M.J. Lightill, Proc. R. Soc. London, Ser. A 211, 564 (1952).

[27] L. Landau and E. Lifchitz, Mécanique des Fluides, 2nd ed. Cours de Physique Théorique, Vol. 6 (Éditions Mir, Moscou, 1989), Sec. 12.

[28] C. Coste, F. Lund, and M. Umeki, Phys. Rev. E 60, 4908 (1999).

[29] J.D. Jackson, Classical Electrodynamics, 2nd ed. (Wiley, New York, 1975).

[30] J.R. Taylor, Scattering Theory: The Quantum Theory on Nonrelativistic Collisions (Wiley, New York, 1972).

[31] P. Roux, J. de Rosny, M. Tanter, and M. Fink, Phys. Rev. Lett. 79, 3170 (1997).

[32] R. Labbé and J.F. Pinton, Phys. Rev. Lett. 81, 1413 (1998).

[33] S. Manneville, J.H. Robres, A. Maurel, P. Petitjeans, and M. Fink, Phys. Fluids 11, 3380 (1999).

[34] M.V. Berry, R.G. Chambers, M.D. Large, C. Upstill, and J.C. Walmsley, Eur. J. Phys. 1, 154 (1980).

[35] Y. Aharonov and D. Bohm, Phys. Rev. 115, 485 (1959).

[36] S. Olariu and I.I. Popescu, Rev. Mod. Phys. 57, 339 (1985).

[37] F. Vivanco, F. Melo, C. Coste, and F. Lund, Phys. Rev. Lett. 10, 1966 (1999).

[38] R. Berthet, S. Fauve, and R. Labbé, Eur. Phys. J. B. (to be published).

[39] It should be noticed that this decomposition is not valid in a narrow cone around the forward direction [36].

[40] P.M. Morse and K.U. Ingard, Theoretical Acoustics (Princeton University Press, Princeton, 1986). 
[41] I.S. Gradshteyn and I.M. Ryzhik, Table of Integrals, Series, and Products, 5th ed. (Academic Press, New York, 1994).

[42] R.G. Newton, Scattering Theory of Waves and Particles, 2nd ed. (Springer-Verlag, New York, 1982).

[43] L. Landau and E. Lifchitz, Mécanique Quantique, 3rd ed. Cours de Physique Théorique, Vol. 3 (Éditions Mir, Moscow, 1988), Sec. 123.

[44] D. Boyer, M. Baffico, and F. Lund, Phys. Fluids 11, 3819 (1999).

[45] V.V. Klimov and V.L. Prozorovskii, Akust. Zh. 33, 128 (1987) [Sov. Phys. Acoust. 1, 79 (1987)].

[46] R. Berthet and F. Lund, Phys. Fluids 7, 2522 (1995).

[47] R. Bernal, C. Coste, F. Lund, and F. Melo, Phys. Rev. Lett. 89, 034501 (2002).

[48] R. Berthet, Ph.D. thesis École Normale Supérieure de Lyon, France, 2001 (unpublished).

[49] R. Berthet, D. Astruc, and S. Fauve (unpublished).

[50] J.H. Ferziger, J. Acoust. Soc. Am. 56, 1705 (1974).

[51] R. Ford and S.G. Llewellyn Smith, J. Fluid Mech. 386, 305 (1999).

[52] A.K. Bose, Nuovo Cimento 32, 679 (1964).

[53] M. Born and E. Wolf, Principles of Optics, 7th ed. (Cambridge University Press, Cambridge, 1999).

[54] W.J. Wiscombe, Appl. Opt. 19, 1505 (1980).
[55] P.R. Gromov, A.B. Ezerskii, and A.L. Fabrikant, Akust. Zh. 28, 763 (1982) [Sov. Phys. Acoust. 28, 452 (1982)].

[56] C. Baudet, S. Ciliberto, and J.F. Pinton, Phys. Rev. Lett. 67, 193 (1991).

[57] A.B. Ezerskii, P.L. Soustov, and V. V Chernov, Acoust. Phys. 46, 670 (2000).

[58] R. Berthet, D. Astruc, and J.L. Estivalézes, in Proceedings of The Sixth AIAA/CEAS Aeroacoustics Conference, 2000 (unpublished).

[59] R. Berthet and D. Astruc (unpublished). (Other details on the numerical simulation can be found in Ref. [48]).

[60] Strictly speaking, the scattered part of the field is defined far from the vortex core. However, the PW method allows the computation of the sound field everywhere, including the nearfield region in the vortex core. In this respect, what we call here $\rho_{\text {scatt }} \equiv \rho_{s}-\rho_{\text {inc }}$ includes the near-field interference pattern. It is an important advantage of the PW approach compared to usual Born approximation, and we hope that the reader will not be confused by this slight misuse of term.

[61] Zhi-Yu Gu and Shang-Wu Qian, J. Phys. A 21, 2573 (1988).

[62] B. Dernoncourt, J-F. Pinton, and S. Fauve, Physica D 117, 181 (1998).

[63] K. Oljaca, X. Gu, A. Glezer, M. Baffico, and F. Lund, Phys. Fluids 10, 886 (1998). 